\title{
Drosophila Glial Glutamate Transporter Eaat1 Is Regulated by Fringe-Mediated Notch Signaling and Is Essential for Larval Locomotion
}

\author{
Stephanie M. Stacey, ${ }^{1 *}$ Nara I. Muraro, ${ }^{4 *}$ Emilie Peco, ${ }^{1}$ Alain Labbé, ${ }^{1}$ Graham B. Thomas, ${ }^{1}$ Richard A. Baines, ${ }^{4}$ \\ and Donald J. van Meyel ${ }^{1,2,3}$ \\ ${ }^{1}$ Centre for Research in Neuroscience and 2 Department of Neurology and Neurosurgery, McGill University, Montreal, Quebec H3G 1A4, ${ }^{3}$ McGill University \\ Health Centre Research Institute, Montreal, Quebec H3H 2R9, Canada, and ${ }^{4}$ Faculty of Life Sciences, University of Manchester, Manchester M13 9PT, \\ United Kingdom
}

In the mammalian CNS, glial cells expressing excitatory amino acid transporters (EAATs) tightly regulate extracellular glutamate levels to control neurotransmission and protect neurons from excitotoxic damage. Dysregulated EAAT expression is associated with several CNS pathologies in humans, yet mechanisms of EAAT regulation and the importance of glutamate transport for CNS development and function in vivo remain incompletely understood. Drosophila is an advanced genetic model with only a single high-affinity glutamate transporter termed Eaat1. We found that Eaat1 expression in CNS glia is regulated by the glycosyltransferase Fringe, which promotes neuron-to-glia signaling through the Delta-Notch ligand-receptor pair during embryogenesis. We made Eaat1 loss-of-function mutations and found that homozygous larvae could not perform the rhythmic peristaltic contractions required for crawling. We found no evidence for excitotoxic cell death or overt defects in the development of neurons and glia, and the crawling defect could be induced by postembryonic inactivation of Eaat1. Eaat1 fully rescued locomotor activity when expressed in only a limited subpopulation of glial cells situated near potential glutamatergic synapses within the CNS neuropil. Eaat1 mutants had deficits in the frequency, amplitude, and kinetics of synaptic currents in motor neurons whose rhythmic patterns of activity may be regulated by glutamatergic neurotransmission among premotor interneurons; similar results were seen with pharmacological manipulations of glutamate transport. Our findings indicate that Eaat 1 expression is promoted by Fringe-mediated neuron-glial communication during development and suggest that Eaat1 plays an essential role in regulating CNS neural circuits that control locomotion in Drosophila.

\section{Introduction}

Glutamate is the principal excitatory neurotransmitter in the mammalian CNS. Extracellular glutamate levels are tightly regulated for precise control of neurotransmission at glutamatergic synapses, and to prevent neuronal cell death from excitotoxicity (Danbolt, 2001). Certain astrocyte populations take up and recycle extracellular glutamate by expressing high-affinity, sodiumdependent excitatory amino acid transporters (EAATs) known as GLAST (alternate name EAAT1) and GLT-1 (alternate name EAAT2), the primary transporters for glutamate in the mamma-

\footnotetext{
Received Feb. 25, 2010; revised Aug. 18, 2010; accepted Sept. 1, 2010.

The work was supported by funds from the Canadian Institutes of Health Research and the Natural Sciences and Engineering Research Council of Canada (Operating Grants to D.J.v.M.), the Research Institute of the McGill University Health Centre and the McGill Faculty of Medicine and (awards to S.M.S., D.J.v.M.), and the Canadian Foundation for Innovation. R.A.B. was supported by the Wellcome Trust. For fly stocks and reagents, we thank Serge Birman, Hermann Aberle, Scott Barolo, Grace Panganiban, Ben Altenhein, Gerd Technau, and Dave 0'Keefe, as well as the Bloomington and Harvard Stock Centers, and the Drosophila Genomic Resource Centre. We also thank Keith Murai, Yong Rao, Kevin Moffatt, Matthias Landgraf, Marc Freeman, Renu Heir, Tiago Ferreira, and members of the Baines and van Meyel laboratories for advice.

*S.M.S. and N.I.M. contributed equally to this work.

Correspondence should be addressed to Donald J. van Meyel, McGill University Health Centre, 1650 Cedar Avenue, L7-221, Montreal, QC H3G 1A4, Canada. E-mail: don.vanmeyel@mcgill.ca.

DOI:10.1523/JNEUROSCI.1021-10.2010

Copyright $\odot 2010$ the authors $\quad$ 0270-6474/10/3014446-12\$15.00/0
}

lian CNS (Matthias et al., 2003). GLAST mutations are found in patients with type 6 episodic ataxia (EA6), a rare form of the disease that variably involves seizures, migraine, cerebellar atrophy, and hemiplegia (Jen et al., 2005; de Vries et al., 2009). Furthermore, expression of EAATs is dysregulated in amyotrophic lateral sclerosis, stroke, epilepsy, schizophrenia, and Alzheimer's and Huntington's diseases, among others (Beart and O'Shea, 2007). However, the mechanisms of EAAT regulation and the consequences of aberrant glutamate transport for CNS function and pathology remain to be fully understood.

Drosophila provides an advanced genetic model system to study EAAT regulation and function in vivo because the differentiation of glial cell subtypes can be studied in an intact nervous system. Moreover, the importance of glutamate transport for CNS development and function can be assessed in vivo with complementary behavioral studies and electrophysiology. In Drosophila, many functional, morphological, and molecular features of glial cells are conserved with mammals (Freeman and Doherty, 2006; Murai and Van Meyel, 2007), including the selective expression of the Eaat1 gene in a subpopulation of glial cells (Soustelle et al., 2002; Freeman et al., 2003). Drosophila Eaat1 has 41 and 35\% amino acid identity, respectively, to human EAAT1 and EAAT2 (Besson et al., 1999). Eaat1 is the only high-affinity 
glutamate transporter in flies (Besson et al., 2000), and so studies of Eaat 1 mutations are unlikely to be complicated by redundancy with the related protein Eaat2, which is a selective high-affinity transporter for taurine and aspartate (Besson et al., 2000, 2005). Previous research in adult flies has shown that reduction of Eaat 1 in glia using RNA interference (RNAi) increases sensitivity to oxidative stress, and results in fewer dopaminergic neurons, degeneration of the brain neuropil, and decreased life span (Rival et al., 2004). However, our understanding of the importance of this glutamate transporter for CNS development and function remains incomplete since RNAi approaches to study Eaat1 function did not reveal phenotypes at embryonic and larval stages (Rival et al., 2004, 2006).

Here, we show that Eaat1 expression in embryogenesis is regulated by the glycosyltransferase Fringe (Fng), which we have shown previously to promote neuron-to-glia signaling through the Delta-Notch ligand-receptor pair (Thomas and van Meyel, 2007). We generated Eaat1 loss-of-function mutations and found that mutant larvae have severe defects of locomotion. Our electrophysiological and genetic approaches provide evidence that Eaat1 acts in a limited subpopulation of CNS glial cells to influence glutamatergic neurotransmission controlling the rhythmic patterning of motor neuron activity. Thus, we have identified cellular and molecular interactions during development that affect the emergence of a functionally distinct glial subtype capable of influencing glutamatergic neurotransmission in the CNS, and discovered an essential role for the Eaat1 glial glutamate transporter in locomotor behavior.

\section{Materials and Methods}

Fly stocks and genetics. Fly stocks were obtained from the Bloomington Stock Center and the Vienna Drosophila RNAi Center (VDRC), or alternative published sources as noted: $P\{$ EPgy2 $\}$ Eaat ${ }^{\text {EY20741 }}$; Df(2L)30A-C (here denoted Eaat1 ${ }^{\text {Df }}$ ); UAS-mCD8::RFP; UAS-Eaat1; UAS-Pros RNAi (VDRC ID 101477); Eaat1-Gal4 (Rival et al., 2004); htl-Gal4 (Shishido et al., 1997); Repo-Gal4 (Sepp and Auld, 2003); tub-Gal80 (McGuire et al., 2003); UAS-N $N^{I C D}$ (Go et al., 1998); UAS-Hairless (Maier et al., 1999); UAS-Tom (Lai et al., 2000); UAS-Eaat1::GFP (Rival et al., 2004); UAS-mCD8::GFP (Lee and Luo, 1999); UAS-nuclearGFP (van Meyel et al., 2003); $S u(H)-l a c Z$ (Griffiths et al., 2007); and $f n g^{L 73}$ and $f n g^{13}$ (Irvine and Wieschaus, 1994; Correia et al., 2003). To create CG31235-nuclear green fluorescent protein (nGFP) and CG31235-Gal4, we PCR amplified 3004 base pairs (bp) of regulatory sequences located immediately upstream of CG31235 using the primers $5^{\prime}$-CATAGTGCATTGGTGAGGTGT- 3 ' and 5'-TACTGGGTGCGCGTTAGGTC-3'. This fragment was inserted into pCR2.1-TOPO and, for CG31235-nGFP, subsequently excised (XbaI/KpnI) and cloned into XbaI/KpnI-digested pH-Stinger (Barolo et al., 2000). For CG31235-Gal4, the fragment was cloned into a modified version of $\mathrm{pH}$ Stinger in which the sequences encoding GFP were replaced by GAL4. Transgenic flies were generated in a $w^{-}$background by standard microinjection procedures (BestGene).

Immunohistochemistry. Embryos and larvae were dissected and fixed according to standard procedures. Monoclonal antibodies obtained from the Developmental Studies Hybridoma Bank included the following: mouse anti-Prospero (dilution 1:25); mouse anti-Fas2 (1:10); mouse anti-Even-skipped (Eve) (dilution 1:10); and mouse anti-Repo (1:50). Other antibodies used were as follows: rabbit antiGFP (1:1000, Invitrogen); rabbit anti- $\beta$-gal (1:1000, MP Biomedicals); mouse anti-glutamine synthetase 2 (Gs2) (1:100, Millipore Bioscience Research Reagents); rabbit anti-cleaved caspase-3 (1:100, Cell Signaling Technology); rabbit anti-VGlut (1:400) (Mahr and Aberle, 2006); and rabbit anti-Nazgul (Naz) (1:500) (von Hilchen et al., 2010). Secondary antibodies used were as follows: Alexa Fluor 488-conjugated goat anti-rabbit and goat anti-mouse (1:300, Invitrogen); Alexa Fluor 647-conjugated goat anti-mouse (1:300, Invitrogen); and Rhodamine Red-X-conjugated goat anti-rabbit and goat anti-mouse (1:300, Jackson ImmunoResearch). Fluorescence-labeled embryos were mounted in Prolong Gold anti-fade medium (Invitrogen). Staining for apoptosis with acridine orange was done as described previously (Arama and Steller, 2006).

In situ hybridization. A $1.7 \mathrm{~kb}$ probe was synthesized from a HindIIIdigested Eaat1 cDNA (RE20434), using a digoxigenin (Dig)-labeling kit (Roche) and T3 RNA polymerase. Prepared embryos (4-20 h after egg laying) were hybridized overnight at $55^{\circ} \mathrm{C}$ using standard procedures, and visualized using anti-Dig-AP (1:1000, Roche). For probe detection in fluorescence in situ hybridization, we used either Fast Red chromogen (Roche) with anti-Dig-AP, or Cy-3-conjugated tyramide reagent (1:50, PerkinElmer) with anti-Dig-POD (1:100, Roche); then, embryos were processed for immunohistochemistry as described above.

Microscopy and imaging. Differential interference contrast microscopy was performed on a Zeiss Axioskop-2 microscope equipped with Nomarski optics. Images were acquired with an AxioCam HRc digital camera and AxioVision software (Zeiss). Confocal microscopy was performed using a Yokogawa spinning disk confocal system (PerkinElmer) and a Nikon Eclipse TE2000-U inverted microscope. $Z$-series images were collected using MetaMorph software (Molecular Devices) and compiled into figures with Adobe Photoshop and Illustrator.

Creation of Eaat1 mutants. Mutations in Eaat1 were generated by imprecise excision of the P-element P\{EPgy2\}Eaat $1^{\text {EY20741 }}$, situated $472 \mathrm{bp}$ upstream of the Eaat 1 start codon. A total of $130 w^{-}$excision lines were screened via PCR (primers: 5' -TTACCAGCATCAAGCTCTCGCTTC3'; 5'-CGCATTCTTCCAATGGTGGTACCT-3'). Two imprecise excisions were identified by amplification of fragments $<2.166 \mathrm{~kb}$, and these fragments were sequenced to determine the extent of the induced deletion and to confirm removal of the Eaat 1 start codon. A precise excision line was also isolated for use as a control.

Reverse transcriptase-PCR. Reverse transcriptase (RT)-PCR was performed on total RNA isolated from first-instar (L1) control $w^{1118}$ and Eaat ${ }^{S M 2 / S M 2}$ mutant larvae (RNEasy Mini Kit and One-Step RT-PCR Kit, Qiagen). The Eaat1 primer set was designed to amplify a band of $448 \mathrm{bp}$ from control $w^{118}$ larvae but not Eaat $1^{\text {SM2/SM2 }}$ mutants (5'-ATTGGTGGACTCGACCTGAG-3'; 5'-CGCTCAGTGTGGTGAAGAAA-3'); the amplicon bridges an intron-exon junction to distinguish cDNA from genomic DNA templates. An Actin5C fragment of $586 \mathrm{bp}$ was amplified as a control $\left(5^{\prime}-\right.$ GAGCGCGGTTACTCTTTCAC-3'; 5' $^{\prime}$ ATCCCGATCCTGATCCTCTT-3').

Semi-quantitative RT-PCR was used to compare Eaat1 expression in $\mathrm{fng}^{13 / L 73}$ mutants to heterozygous controls (genotype: $\mathrm{fng}^{13} / \mathrm{TM} 3$, twiGAL4,UAS-GFP). Briefly, total RNA was isolated from pools of $\sim 80 \mathrm{~L} 1$ larvae (RNEasy Plus Mini Kit, Qiagen), then $1 \mu \mathrm{g}$ was used as a template for cDNA synthesis with M-MuLV RT (New England Biolabs), oligo-dT primers, and random hexamers. PCR was then performed with Platinum Taq (Invitrogen) and either the Eaat1 or Actin5C primers above, using 1 $\mu \mathrm{l}$ of cDNA as a template in $25 \mu \mathrm{l}$ reactions. Paired samples from controls and mutants were removed from the thermal cycler at each cycle from 21 through 27 , and immediately incubated at $72^{\circ} \mathrm{C}$ for $10 \mathrm{~min}$. Samples were run on a $1 \%$ agar gel stained with ethidium bromide, and the measured intensity of the Eaat1 PCR product was normalized to that of Actin-5C for each of cycles 21 through 27, using ImageJ software to analyze nonsaturated images.

Behavior assays. Studies of mechanosensory performance and larval crawling were done as described previously (Kernan et al., 1994; Carhan et al., 2004) on L1 larvae selected $0-2 \mathrm{~h}$ posthatching and placed on $2.3 \%$ agar plates (room temperature). For each assay, the animals were allowed to acclimatize for $5 \mathrm{~min}$. Before and after crawling, each animal was touched lightly to ensure they could respond to mechanical stimulation. For each trial, the number of full-body peristaltic contractions (forward or backward) was counted during a $3 \mathrm{~min}$ period. Three consecutive trials were performed for each larva, and these were averaged to produce a single data point. For each genotype, 15-40 larvae were examined. Heterozygous controls were generated by crossing Eaat 1 alleles to $w^{1118}$ flies.

To assess the developmental or acute requirement for Eaat1 in larval locomotion, a temperature-sensitive Gal80 allele $\left(G a l 80^{t s}\right)$ was used to repress Gal4-mediated rescue of Eaat $1^{\text {SM2/SM2 }}$ mutants at larval stages (McGuire et al., 2003). Gal80 ${ }^{\text {ts }}$ binds and represses Gal 4 activity at $18^{\circ} \mathrm{C}$, 
but at $29^{\circ} \mathrm{C}$ it is nonfunctional. The broadly expressed tubulin-Gal $80^{t s}$ (tubP-Gal80ts) was added into the background of a rescue (genotype: Eaat1 $^{\text {SM2 }}$, CG31235-Gal4; UAS-Eaat1::GFP/tub-Gal80 ${ }^{\text {ts }}$ ). Embryos were collected and raised at $29^{\circ} \mathrm{C}$ to inactivate $\mathrm{Gal} 80^{\text {ts }}$ and allow sufficient expression of the UAS-Eaat $1:$ GFP transgene to rescue throughout embryogenesis. Upon hatching $(0 \mathrm{~h})$, larvae were assayed for full-body peristaltic contraction rates as described above, then shifted to $18^{\circ} \mathrm{C}$ to repress UAS-Eaat 1::GFP expression for the duration of the experiment. Larval contraction rates were again assessed at 30, 40, 44, and $48 \mathrm{~h}$ following the temperature shift to $18^{\circ} \mathrm{C}$. Control animals were of the same genotype but lacked tubP-Gal80ts. To examine levels of Eaat1::GFP expression over the course of this experiment, larvae at 0 and $48 \mathrm{~h}$ were assessed by anti-GFP immunohistochemistry.

Electrophysiology. Whole-cell voltage-clamp recordings were performed at room temperature $\left(22-24^{\circ} \mathrm{C}\right)$ in dissected $\mathrm{L} 1$ larvae, $1-4 \mathrm{~h}$ after hatching, using thick-walled borosilicate glass electrodes (GC100F10; Harvard Apparatus) fire polished to resistances of 15-20 M $\Omega$. External saline composition was as follows: $135 \mathrm{~mm} \mathrm{NaCl}, 5 \mathrm{~mm} \mathrm{KCl}, 4 \mathrm{~mm}$ $\mathrm{MgCl}_{2} \cdot 6 \mathrm{H}_{2} \mathrm{O}, 2 \mathrm{mM} \mathrm{CaCl}_{2}, 5 \mathrm{~mm} \mathrm{~N}$-Tris(hydroxymethyl)methyl-2aminoethanesulfonic acid and $36 \mathrm{~mm}$ sucrose, $\mathrm{pH}$ 7.15. The internal patch solution was as follows: $140 \mathrm{~mm} \mathrm{KCH}_{3} \mathrm{SO}_{3}, 2 \mathrm{~mm} \mathrm{MgCl} 2,2 \mathrm{~mm}$ EGTA, $2 \mathrm{~mm} \mathrm{KCl}$, and $20 \mathrm{~mm}$ HEPES, pH 7.4. Central neurons were visualized and accessed for electrophysiology using an Olympus BX51W1 microscope and 60× water-immersion lens combined with Nomarski optics, (Baines and Bate, 1998). RP2 and aCC motor neurons were initially identified by their size and location; after recording, their identity was confirmed (on the basis of morphology and axon trajectory) by labeling with $0.1 \%$ Alexa Fluor 488 hydrazide (Invitrogen), which was included in the internal patch solution. Recordings were made using a Multiclamp 700B amplifier controlled by pClamp 9.2 via a Digidata 1322A analog-to-digital converter [Molecular Devices (MDS Analytical Technologies)]. Only cells with input resistance $>1 \mathrm{G} \Omega$ were accepted for analysis. After breakthrough, currents were measured for $3 \mathrm{~min}$. If no currents were recorded in this period, the cell was termed "silent." Traces were sampled at $20 \mathrm{kHz}$ and filtered at $2 \mathrm{kHz}$ low pass. Amplitudes of spontaneous rhythmic currents (SRCs) were measured using Minianalysis 6.0.3 (Synaptosoft). Currents shown were normalized for cell capacitance (determined by integration of the area under the capacitive transients for the average of 10 steps from -60 to $-90 \mathrm{mV}$ ). SRCs showing multiple peaks were quantified as a single event; the amplitude was taken from the largest peak, which was the first one in nearly every instance. For pharmacology experiments, a $100 \mathrm{~mm}$ stock solution of DL-TBOA (Tocris Bioscience) was used to prepare a working solution at $300 \mu \mathrm{M}$ in external saline. Saline bathing the preparation during larval dissection was exchanged for this working solution of TBOA before recording.

Statistics. When comparing two groups, data were analyzed using either a paired or unpaired $t$ test (two-tailed, Mann-Whitney) as appropriate. When comparing more than two groups, we used one-way ANOVA. For ANOVA, the Kruskal-Wallis test with Dunn's post hoc comparisons was used if the data in one or more of the groups were not normally distributed, as determined by the Shapiro-Wilk test. If data in all groups were normally distributed, one-way ANOVA with Dunnett's test was used to compare the groups. Values shown are mean \pm SEM.

\section{Results}

\section{Eaat1 expression in Drosophila CNS}

The major nerve tracts of the Drosophila ventral nerve cord (VNC), called commissures and longitudinal connectives, mark a dense neuropil of axon projections, dendrites, and synapses within the segmentally repeated embryonic and larval CNS (Fig. $1 A, B)$. A subset of CNS glial cells expresses the gene CG31235 (supplemental Fig. S1, available at www.jneurosci.org as supplemental material), including the nine longitudinal glia (LG) found in each VNC hemi-segment. LG lie just dorsal to the longitudinal connectives and ensheath the neuropil (Jacobs et al., 1989; Stacey et al., 2007). In building genetic tools to study these glia in vivo, we found that a $3 \mathrm{~kb}$ promoter/enhancer of CG31235 can direct the expression of Gal4 (Fig. $1 B^{\prime}$ ) or nuclear GFP (nGFP) (Fig. $1 C^{\prime}$ ) transgenes to the nine LG (Beckervordersandforth et al., 2008), plus five additional glial cells in each VNC hemi-segment (supplemental Figs. S2, S3, available at www.jneurosci.org as supplemental material). We used in situ hybridization to examine the expression of Eaat1 transcripts in the VNC of CG31235-nGFP animals (Fig. $1 C-\mathrm{C}^{\prime \prime}$ ) and noted that Eaat1 was expressed in glial cells, including a subset of LG (Fig. 1D). Onset of Eaat1 transcript expression occurred rather late in embryogenesis (stages 15-16), which is consistent with previous reports (Besson et al., 1999; Soustelle et al., 2002), and only narrowly precedes the initiation of spontaneous and uncoordinated muscle contractions (Crisp et al., 2008).

Using Eaat1-Gal4 to mark Eaat1-expressing cells in the VNC (supplemental Fig. S3, available at www.jneurosci.org as supplemental material), we found that virtually all of them also expressed CG31235-nGFP (423/430 cells counted). The nine LG in each hemi-segment can be subdivided further because the anterior-most six of these cells express the transcription factor Prospero (Pros) (Thomas and van Meyel, 2007). We found that $84 \%$ (173/205) of Eaat1-Gal4 cells are also Pros positive (supplemental Fig. S3, available at www.jneurosci.org as supplemental material), indicating that a large majority of Eaat1-expressing cells are of the anterior LG subtype. This subtype also expresses Gs2 (Freeman et al., 2003; Thomas and van Meyel, 2007). Glutamine synthetases convert glutamate to glutamine, which is synaptically inert and can be safely recycled back to neurons (Hertz et al., 1999). Coexpression of Gs2 and Eaat1 in the anterior LG strongly suggests that this subtype of glial cell is well equipped for the uptake and metabolism of glutamate from CNS synapses in Drosophila, and could potentially modulate glutamatergic neurotransmission. Consistent with this, the presynaptic vesicular glutamate transporter VGlut (Mahr and Aberle, 2006; Daniels et al., 2008), and the postsynaptic glutamate receptor KaiRIA (GluRIID) (Featherstone et al., 2005) are both expressed in the dorsal neuropil of the VNC of embryos and larvae, near the cell bodies of LG. To determine whether Eaat1-expressing LG infiltrate the neuropil and express Eaat1 near putative glutamatergic synapses in first instar (L1) larvae, we used Eaat1-Gal4 to drive expression of an Eaat1::GFP fusion protein (UAS-Eaat1::GFP) (Fig. 1 E; supplemental Fig. S4, available at www.jneurosci.org as supplemental material), and colabeled with either the membranetargeted reporter mCD8-red fluorescent protein (RFP) (supplemental Fig. S4, available at www.jneurosci.org as supplemental material) or anti-VGlut (Fig. $1 E^{\prime}$ ) to mark potential sites of glutamatergic presynaptic terminals in first instar larvae. Eaat 1::GFP was broadly expressed among the RFP-labeled glial membranes and, relative to RFP, appeared to be enriched in glial membranes that had infiltrated the CNS neuropil (supplemental Fig. S4, available at www.jneurosci.org as supplemental material). VGlut-positive puncta were located dorsally within the VNC neuropil (Fig. $1 E^{\prime \prime}$ ), similar to the pattern observed previously in third instar larvae (Daniels et al., 2008). Optical sections through the neuropil revealed extensive Eaat 1::GFP labeling in close proximity to VGlut-positive puncta (Fig. $1 F, G$ ), consistent with the idea that glutamatergic transmission at CNS synapses in Drosophila could be influenced by the Pros-positive anterior LG subtype that express both the glutamate transporter Eaat1 and the glutamine synthetase Gs2. 

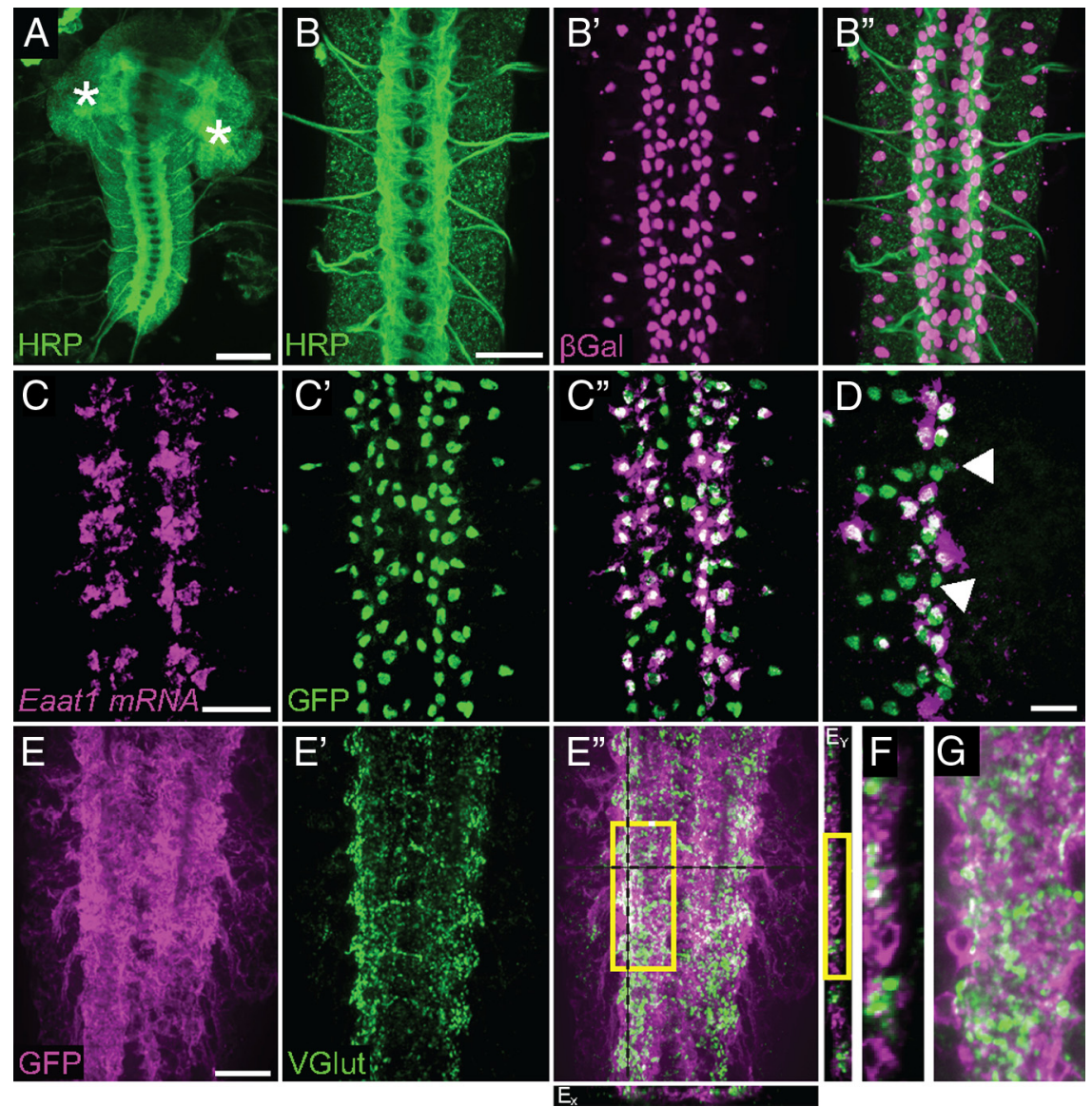

Figure 1. Eaat1 is expressed in a subset of CNS glia. $\boldsymbol{A}-\boldsymbol{B}^{\prime \prime}$, Dorsal view of the CNS of a filleted embryo at stages $16-17$ (genotype: (G31235-Gal4/UAS-nuclear-lacZ; (G31235-Gal4/+). A, Anti-HRP (green) labels all neurons, and reveals the brain lobes (asterisks) and the major axon tracts of the VNC. Scale bar, $50 \mu \mathrm{m}$. $\boldsymbol{B}-\boldsymbol{B}^{\prime \prime}$, Higher magnification of the same genotype to highlight five segments of the VNC. $\boldsymbol{B}$, Anti-HRP (green) reveals the ladder-like organization of commissures and longitudinal connectives. Scale bar, $20 \mu \mathrm{m} . \boldsymbol{B}^{\prime}$, CG31235-Gal4-expressing glia revealed with anti- $\beta$-galactosidase staining (magenta). $\boldsymbol{B}^{\prime \prime}$, Overlay of $\boldsymbol{B}$ and $\boldsymbol{B}^{\prime}$. Based on cell number, position, and colabeling with other markers (supplemental Figs. S2, S3, available at www.jneurosci.org as supplemental material), the (G31235-Gal4-positive subpopulation of glia in the VNC is likely composed of all nine $L G$, all three cell body glia (MM-CBG, M-CBG, and L-CBG), plus two intersegmental nerve glia (M-ISNG, L-ISNG). C-C", Five segments of the VNC of a CG31235-nuclear-GFP embryo at stage 16. C, Whole-mount fluorescence in situ hybridization for Eaat1 (magenta). $\boldsymbol{C}^{\prime}$, CG31235-nGFP glia (green). Scale bar, $20 \mu \mathrm{m}$. $\boldsymbol{C}^{\prime \prime}$, Overlay of $\mathbf{C}$ and $\boldsymbol{C}^{\prime}$. A subset of LG coexpress Eaat1 and CG31235-nGFP. D, Embryo of same genotype and age at higher magnification, showing only three hemi-segments. Arrowheads indicate several CG31235-positive glia that do not express Eaat1. Scale bar, $10 \mu \mathrm{m}$. E-G, A single optical slice showing five segments of the VNC of a $L 1$ larva 0 -2 $\mathrm{h}$ posthatching (genotype: Eaat1-Gal4/+ ; UAS-Eaat1::GFP/+). E, Eaat1::GFP (magenta) is extensively localized to Eaat1 glial membrane projections within the VNC. Scale bar, $20 \mu \mathrm{m}$. $\boldsymbol{E}^{\prime}$, Staining with anti-VGlut (green), a marker for presynaptic glutamatergic terminals, to reveal potential sites of glutamatergic synapses. VGlut staining is punctate and segmentally repeated. $\boldsymbol{E}^{\prime \prime}$, Overlay of $\boldsymbol{E}$ and $\left.\boldsymbol{E}^{\prime}\right)$. $\boldsymbol{E}_{\gamma^{\prime}}$ Longitudinal section of VNC at position indicated by black dotted vertical black line. Eaat1::GFP (magenta) is observed immediately adjacent to VGlut (green) puncta. $\boldsymbol{E}_{X^{\prime}}$ Cross section of VNC at position indicated by dotted horizontal black line. $\boldsymbol{F}$, Close-up view of yellow boxed area in $\boldsymbol{E}_{\boldsymbol{Y}}$. $\boldsymbol{G}$, Close-up view of yellow boxed area in $\boldsymbol{E}^{\prime \prime}$. Anterior is at the top in all panels of all figures.

\section{Regulation of Eaat1 by Fringe-mediated Notch signaling}

Activated Notch signaling in the Pros-expressing anterior LG of wild-type (WT) embryos can be visualized directly with the Notchsensitive reporter Su(H)-lacZ (Fig. 2A-A' ) (Griffiths et al., 2007). We have found previously that the glycosyltransferase Fng is expressed in the anterior LG subtype, where it sensitizes the Notch receptor to signaling induced by its neuron-derived ligand Delta and thereby promotes the expression of Pros (Thomas and van Meyel, 2007). To determine whether Eaat1 expression in LG was also regulated by Fng-mediated Notch signaling, we performed two experiments; first, we examined $f n g^{13 / L 73}$ mutants (Fig. $2 C$ ), and second, we overexpressed the Notch antagonist Hairless (Fig. 2D), a transcrip- tional repressor specific for the Notch pathway (Morel et al., 2001). We have shown previously that LG are neither missing nor improperly specified under either of these experimental conditions (Thomas and van Meyel, 2007). We observed that Eaat1 expression in the anterior LG subtype is severely reduced in fing mutant embryos; this is likely due to a failure to elevate Notch signaling in these cells, since overexpression of Hairless had the same effect.

We also used semi-quantitative RTPCR to estimate the relative level of Eaat1 expression between fng mutants and controls in newly hatched L1 larvae. Normalized Eaat1 levels in $\mathrm{fng}^{13 / L 73}$ mutants were reduced to $27-64 \%$ of $f_{n} g^{13 /+}$ heterozygous controls (Fig. $2 E$ ). Thus, by controlling the expression of Eaat1, Fng promotes terminal differentiation of a glial cell subtype that can take up glutamate and perhaps modulate neurotransmission.

To investigate whether Fng acts via Pros to promote the expression of Eaat1 in anterior LG, we used RNAi interference and found that Eaat1 transcript levels remained present in LG lacking detectable levels of Pros (supplemental Fig. S5, available at www.jneurosci.org as supplemental material). This result suggests that Pros is unlikely to promote the expression of Eaat1 at embryonic stages, and that Fng is likely to regulate Eaat1 and Pros independently. Second, we examined wild-type animals and found that Pros is normally exported from anterior LG and degraded at late stages of embryogenesis (supplemental Fig. S5, available at www. jneurosci.org as supplemental material). Therefore, Pros is unlikely to be important for the maintenance of Eaat1 in the anterior LG in L1 larvae.

\section{Creation of Eaat1 mutations in Drosophila}

To observe the consequences of removing Eaat1 in vivo, we created Eaat1 null mutations by imprecise excision of a $\mathrm{P}$ element $\left(P\{\right.$ EPgy 2$\}$ Eaat $\left.1^{\text {EY20741 }}\right)$ inserted $472 \mathrm{bp}$ upstream of the Eaat1 start codon (Fig. $3 A$ ). Using PCR and sequencing, two novel deletion mutations were identified and confirmed to remove the start codon of Eaat 1 (Fig. 3A-C). The allele Eaat $1^{S M 1}$ was found to remove 841 bp of the Eaat1 gene, while Eaat ${ }^{S M 2}$ removed 1293 bp (Fig. 3B). If translated, both mutations would fail to encode the first two transmembrane domains of the Eaat 1 transporter, with a portion of the third transmembrane domain also missing in Eaat $1^{S M 2}$. Since both mutations remove the start codon and multiple transmembrane domains, they are predicted to severely disrupt the properties of Eaat1. To determine whether Eaat 1 mRNA is lost due to Eaat ${ }^{S M 2}$ mutation, we used RT-PCR to compare Eaat 1 transcripts between control (WT) and Eaat $1^{S M 2 / S M 2}$ larvae at L1 
(Fig. 3C). Robust amplification of an Eaat1-specific RT-PCR product was observed in WT larvae but not Eaat ${ }^{\text {SM2/SM2 }}$ mutants, confirming loss of Eaat1 expression.

\section{Severe locomotor dysfunction in Eaat1 mutants}

Mutant L1 larvae hatched at a frequency of $26 \%$ from an intercross of balanced, heterozygous parents, but they were $1-2 \mathrm{~h}$ slower to emerge from the eggshell and had severely compromised motor function. Drosophila larvae normally move in a forward direction by peristaltic crawling, with rhythmic and coordinated waves of muscle contraction passing along the body wall segments in a posterior-toanterior direction. Larvae homozygous for either Eaat ${ }^{S M 1}$ or Eaat $1^{S M 2}$ seldom made full-body peristaltic contractions (Fig. 3D). They had long periods of inactivity that were only occasionally interrupted by attempted contractions that were often slow and incomplete, and left the animal contorted (supplemental Movies 1, 2, available at www.jneurosci.org as supplemental material).

We quantified the number of full-body peristaltic contractions made during $3 \mathrm{~min}$ trials and found that Eaat $1^{\text {SM2/+ }}$ heterozygous controls made an average of $89.1 \pm 6.0$ contractions (mean \pm SEM) (Fig. 3D). In contrast, the contraction rates in Eaat $1^{S M 1 / S M 1}$ and Eaat $1^{\text {SM2/SM2 }}$ mutants, as well as Eaat $1^{\text {SM2/Df }}$ hemizygotes, were dramatically reduced, as follows: $1.8 \pm 0.3,0.7 \pm 0.5$, and $1.7 \pm 0.4$ contractions per $3 \mathrm{~min}$, respectively. Eaat $1^{S M 2 /+}$ heterozygotes had twice the rate of contractions as larvae heterozygous for a precise excision of $P\{$ EPgy 2$\}$ Eaat $^{E Y 20741}(45.2 \pm 3.0)$, but this was not significant in the nonparametric ANOVA used to analyze our complete dataset. Importantly, the severe crawling impairment that we quantified in the mutants supports the idea that both Eaat $1^{S M 1}$ and Eaat $1^{S M 2}$ are null alleles; the effects of Eaat $1^{S M 1 / S M 1}$ and Eaat $1^{S M 2 / S M 2}$ were similar, and the effects in Eaat $1^{S M 2 / S M 2}$ are as severe as Eaat $1^{\text {SM2/Df }}$.

We then measured the behavior of mutant larvae relative to heterozygous controls in an established mechanosensation assay where withdrawal, turning, and evasive crawling are scored in response to light touch (Kernan et al., 1994). Notably, all mutant larvae were sensitive to touch and withdrew, as did controls, indicating that they did not have a generalized paralysis. However, their score in this assay was reduced because they could not reliably perform the reverse or forward peristaltic contractions that typically allow escape (Fig. 3E; supplemental Movies 1,2 , available at www.jneurosci.org as supplemental material).

$E$
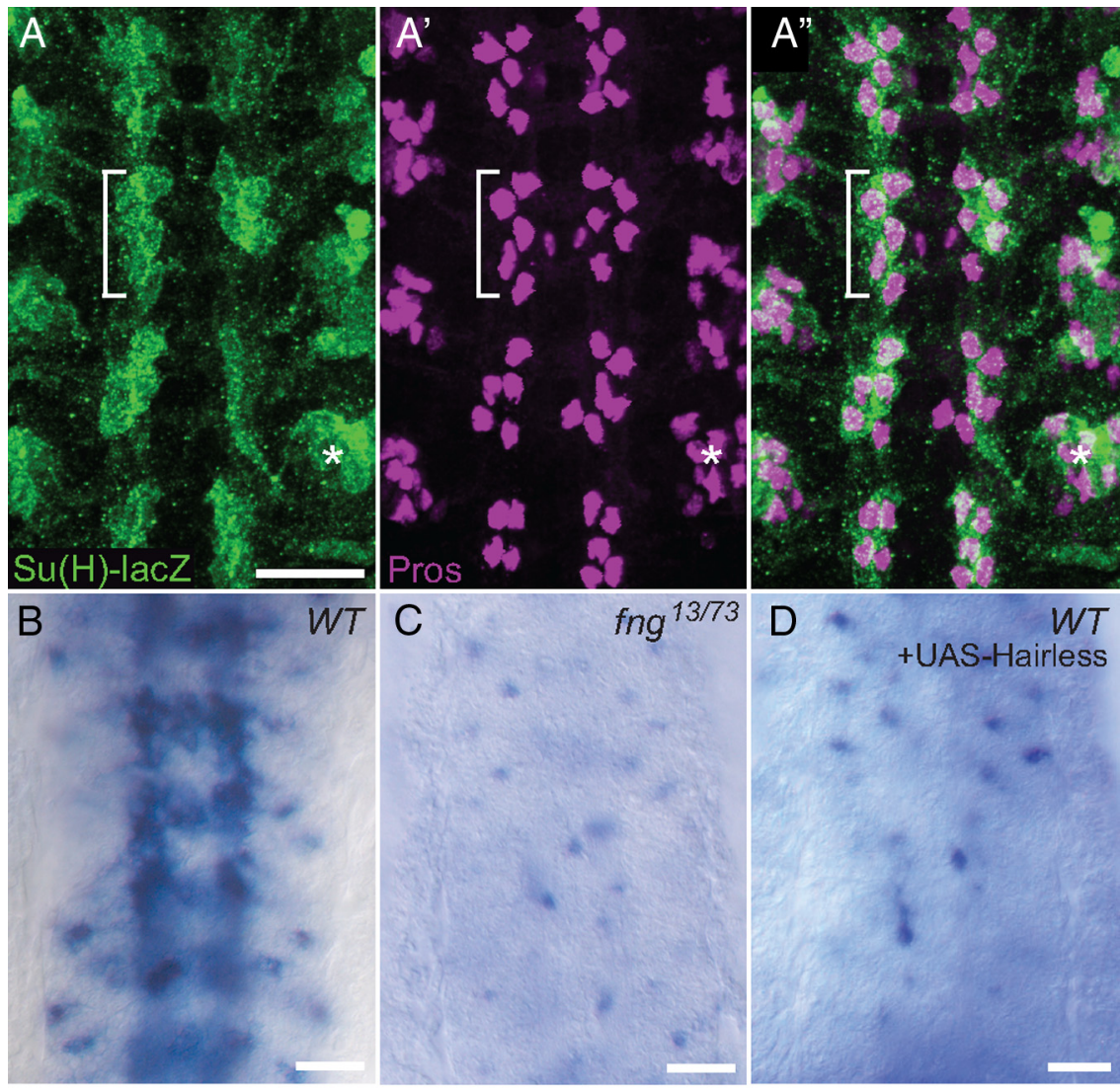

WT

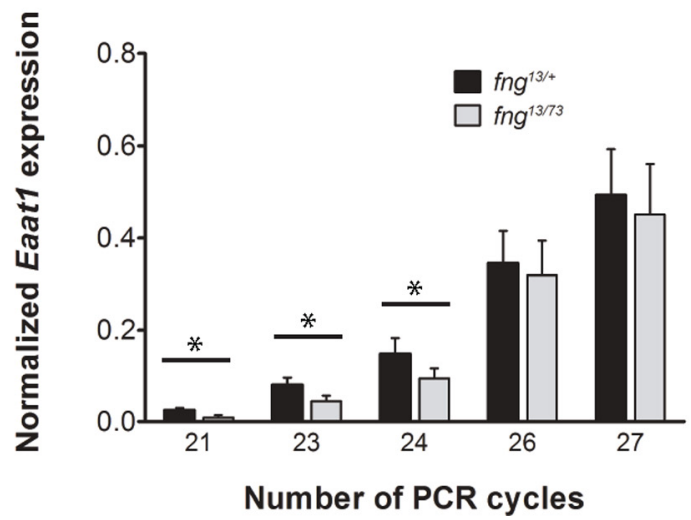

Figure 2. Fng is required for Eaat1 expression in the anterior $L G . A-A^{\prime \prime}$, Four segments of the VNC of stage 16 embryo expressing $\mathrm{Su}(\boldsymbol{H})$-lacZ, a Notch-responsive reporter. A white bracket notes where anti- $\beta$-galactosidase staining for $\mathrm{Su}(\boldsymbol{H})$-lacZ (A, green) overlaps with the Pros-expressing anterior LG ( $\boldsymbol{A}^{\prime}$, magenta) in one hemi-segment. The white asterisk notes one cluster of Pros-positive neurons positioned laterally in each hemi-segment. Scale bar, $10 \mu \mathrm{m} . \boldsymbol{A}^{\prime \prime}$, Overlay of $\boldsymbol{A}$ and $\boldsymbol{A}^{\prime}$. $\boldsymbol{B}-\boldsymbol{D}$, All panels show whole-mount in situ hybridization staining for Eaat1 in the VNC at embryonic stages $16-17$. Scale bars, $10 \mu \mathrm{m}$. B, Eaat 1 transcript is strongly expressed in a subset of LG in WT and control embryos (genotype shown: $h t-G a l 4 /+$ ). C, fng ${ }^{13} / \mathrm{fng}^{73}$ mutants have dramatically reduced Eaat1 levels. D, Expression of the Notch antagonist Hairless in LG (genotype: htl-Gal4/UAS-Hairless) also reduces Eaat1 expression. Anterior is at the top in all panels of all figures. E, F, Plot of semi-quantitative RT-PCR comparing Eaat 1 expression (normalized to Actin-5C) between $\mathrm{fng}^{13} / \mathrm{TM} 3$ heterozygous controls and $f n g^{13 / 273}$ mutants. Each bar represents the mean \pm SEM of four distinct trials. At 21, 23, and 24 cycles (when PCR amplification still appears to be linear), the mutants had an average of 27,55 , and $64 \%$ the level of controls, respectively. Asterisks indicate significant differences at $p<0.05$ (paired $t$ test) for comparisons of controls and fng mutants. At cycles 26 and 27 , the difference between the two groups was not significant, likely due to nonlinear amplification.

The locomotor defects of Eaat $1^{S M 2 / S M 2}$ mutants were fully rescued with a UAS-Eaat1 transgene driven by two copies of CG31235-Gal4. Both the manner and frequency of the full-body contractions were restored to control levels $(74.9 \pm 10.3$ per 3 min) (Fig. 3D). This demonstrates the specificity of the phenotype for Eaat1 and refines the requirement for Eaat1 in larval 


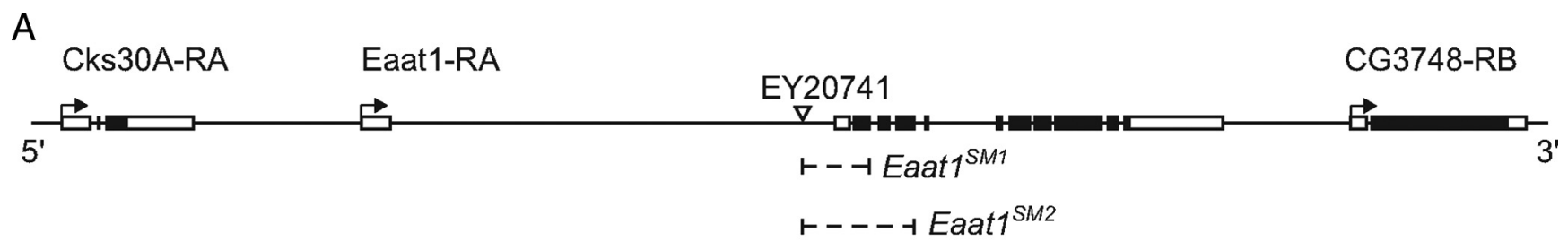

B

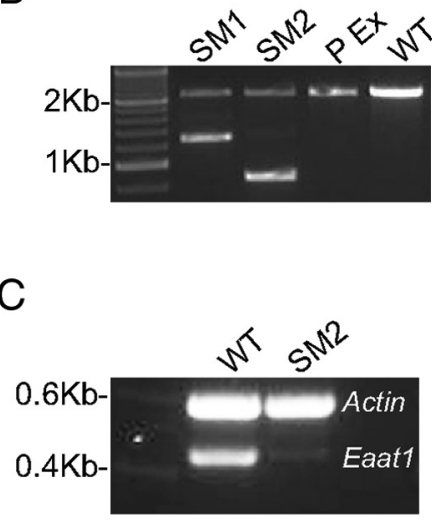

$\mathrm{F}$

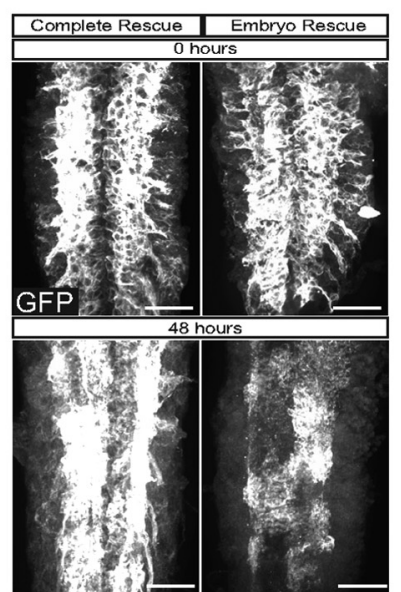

D

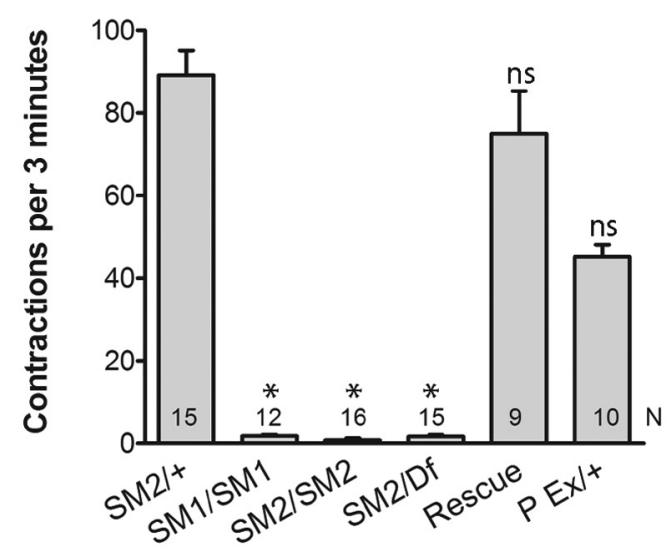

E

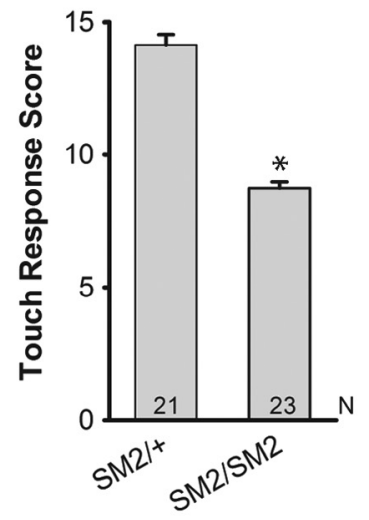

G

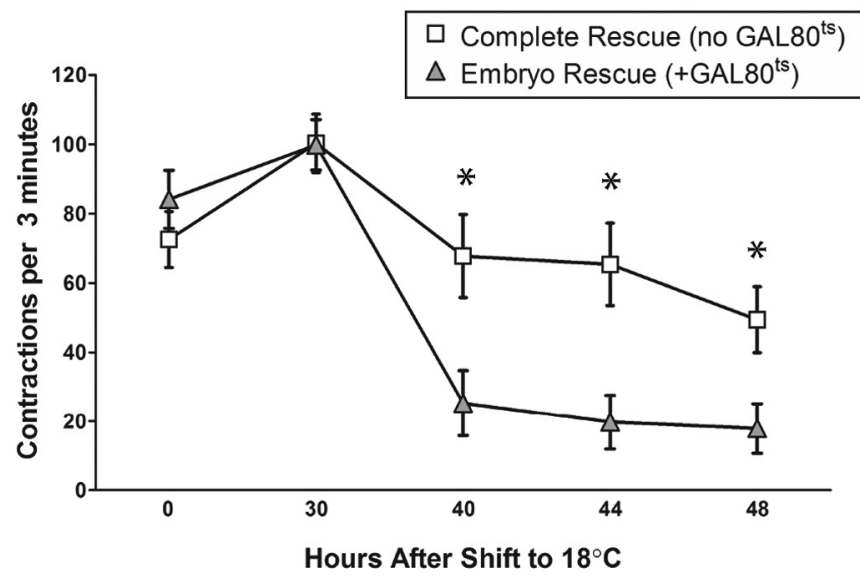

Figure 3. Generation of Eaat 1 mutants and characterization of crawling defects. $A$, The Eaat 7 locus and neighboring genes Cks30A and CG3748, and the extent of the induced deletion mutations Eaat $^{\text {SM1 } 1}$ and Eaat $7^{\text {SM2 }}$ generated by imprecise excision of EY20741 (inverted triangle). Eaat1-RA (FlyBase) encodes full-length Eaat1 corresponding to EST RE20434. Coding exons are in black, noncoding exons are in white. $\boldsymbol{B}$, Eaat $7^{S M 1}$ and Eaat $7^{S M 2}$ were identified via PCR screening. WT animals and precise excisions (P Ex) of EY20741 generated a $2.166 \mathrm{~kb} P C R$ fragment, while imprecise excisions were identified by amplification of shorter fragments with the same primers. DNA sequencing of these shorter fragments revealed that Eaat $7^{\text {SM1 }}$ deleted 841 bp and Eaat ${ }^{\text {SM2 }}$ removed 1293 bp, both to the right of the original insertion site, as indicated by dotted lines in A.C, RT-PCR of WT and Eaat $1^{S M 2}$ mutants shows a strong Actin5C control band in both genotypes. A strong Eaat 1 band is present in the WT but negligible in Eaat ${ }^{\text {SM2 }}$. D, Eaat1 mutant larvae (L1, $0-2 \mathrm{~h}$ posthatching) show dramatically reduced numbers of peristaltic contractions. Bars show mean \pm SEM for Eaat 1 heterozygotes $\left(\right.$ Eaat $\left.1^{\text {SM2 }} /+\right)$, homozygotes (Eaat ${ }^{\text {SM1 }}$ and Eaat ${ }^{\text {SM2 }}$ ), hemizygotes (Eaat $\left.7^{\text {SM2 }} / D f\right)$, rescued animals (CG31235-Gal4, Eaat $7^{\text {SM2 }}$; UAS-Eaat $\left.1 /+\right)$, and controls heterozygous for the precise excision of EY20741 (P Ex/+). Expression of UAS-Eaat1 with (G31235-Gal4 is sufficient to rescue Eaat ${ }^{\text {SM2 }}$ larval peristaltic contraction rates to control levels. Asterisks indicate significance

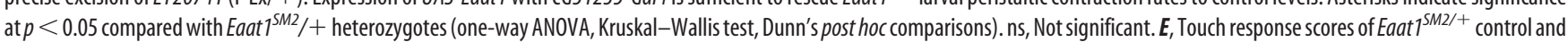
Eaat $^{\text {SM2/SM2 }}$ larvae (Kernan et al., 1994). Bars show mean \pm SEM. Each larva was tested four times, giving a score of 16 . The number ( $(N)$ of larvae tested is indicated for each genotype. Asterisk indicates significance at $p<0.0001$ compared with Eaat ${ }^{5 M 2} /+$ control ( $t$ test, two-tailed, Mann-Whitney). $\boldsymbol{F}, \mathbf{G}$, Galso ${ }^{\text {ts }}$-dependent withdrawal of Eaat1::GFP rescue following completion of embryogenesis. F, GFP expression in larval VNCs at hatching and $48 \mathrm{~h}$ following the shift to $18^{\circ} \mathrm{C}$. At hatching, the levels of Eaat 1::GFP are similar between the Complete Rescue (genotype: Eaat ${ }^{\text {SM2 }}$, CG31235-Gal4;UAS-Eaat1::GFP/+) and Embryo Rescue (genotype: Eaat T ${ }^{\text {SM2 }}$, (G31235-Gal4;UAS-Eaat1::GFP/tub-Gal80 ${ }^{\text {ts }}$ ) groups. At 48 h, Eaat1::GFP expression is markedly decreased in the Embryo Rescue group. Scale bars, $20 \mu \mathrm{m}$. G, Plot of contraction rates (mean \pm SEM) for Complete Rescue (white squares) and Embryo Rescue (gray triangles). Fifteen or more larvae were examined for each plotted mean. Asterisks indicate significant differences at $p<0.01$ ( $t$ test, two-tailed, Mann-Whitney) for comparisons between Complete Rescue and Embryo Rescue at each time point.

locomotion to the subset of CNS glia that expresses CG31235Gal4. Two additional glial-specific Gal4 drivers were also tested for their ability to rescue Eaat $1^{\text {SM2/SM2 }}$ mutants: Eaat1-Gal4 (Rival et al., 2004) and Repo-Gal4 (Sepp and Auld, 2003), which is expressed more broadly in all lateral glia. Like CG31235-Gal4, these drivers also rescued larval contractions (data not shown). In additional observations, we noted that Eaat ${ }^{S M 1}$ and Eaat1 ${ }^{S M 2}$ mutations caused lethality before pupation, whether homozygous, hemizygous over Eaat $1^{D f}$, or in transallelic combination with one another. Mutant larvae all died by $48 \mathrm{~h}$ posthatching. Though both CG31235-Gal4 and Eaat1-Gal4 rescued larval contractions, neither rescued the viability of mutant animals beyond third instar. However, Repo-Gal4 rescued larval contractions, and these animals survived to adulthood, suggesting 
that expression of Eaat1 in a broader number of glial cells is required for the animals to progress through pupation and eclosion.

The locomotor defect in Eaat1 mutants could be secondary to developmental defects arising during embryogenesis or could result from acute lack of Eaat1 function during larval locomotion. To address this directly, we rescued Eaatl function in Eaat1mutants through embryonic stages (with CG31235-Gal4 and UAS-Eaat 1::GFP), then used a temperature-sensitive repressor of Gal4 $\left(\mathrm{Gal} 80^{\mathrm{ts}}\right)$ to inhibit the expression of the UAS-Eaat1::GFP transgene and thereby discontinue the rescue in newly hatched L1 larvae (McGuire et al., 2003). To do this, we collected control and experimental embryos at $29^{\circ} \mathrm{C}$, but switched them to $18^{\circ} \mathrm{C}$ immediately after hatching and raised them at $18^{\circ} \mathrm{C}$ thereafter. The control group (hereafter called Complete Rescue) was Eaat1::GFP-rescued mutants lacking tubP-Gal80 ${ }^{t s}$ (genotype: Eaat1 $^{\text {SM2/SM2 }}$, CG31235-Gal4; UAS-Eaat1::GFP/+). The genotype of the experimental group (Embryo Rescue) was Eaat $1^{S M 2 / S M 2}$, CG31235-Gal4; UAS-Eaat1::GFP/tubP-Gal80 ${ }^{\text {ts }}$. We used GFP fluorescence to measure the expression of Eaat1::GFP in the Complete Rescue control and the Embryo Rescue groups. At hatching, both groups had similar levels of GFP expression in the VNC (Fig. 3F). However, by $48 \mathrm{~h}$ at $18^{\circ} \mathrm{C}$, there was a marked decrease in GFP expression in the Embryo Rescue larvae compared with the Complete Rescue larvae (Fig. 3F), likely reflecting the degradation of Eaat 1::GFP produced at embryonic stages, and reduced synthesis of new Eaat1::GFP in Gal80 ${ }^{t s}$-expressing larvae.

Before proceeding, we studied whether simply raising larvae at $18^{\circ} \mathrm{C}$ shift influenced crawling behavior by measuring contraction rates among cohorts of control Eaat $1^{\text {SM2/+ }}$ heterozygotes at 0,30 , and $48 \mathrm{~h}$ following the shift to $18^{\circ} \mathrm{C}$; these measured $110.6 \pm 8.1(N=17), 110.9 \pm 8.8(N=17)$, and $102.7 \pm 9.7$ $(N=15)$, respectively, and confirmed that there was no effect of the temperature shift alone.

We then quantified larval contraction rates in the Complete Rescue versus Embryo Rescue groups at hatching $(0 \mathrm{~h})$ and up to $48 \mathrm{~h}$ after the shift to $18^{\circ} \mathrm{C}$ (Fig. $3 G$ ). Average contraction rates for Complete Rescue controls (Fig. 3G, white squares) and Embryo Rescue animals (Fig. 3G, gray triangles) were similar at 0 and $30 \mathrm{~h}$. However, at 40, 44, and $48 \mathrm{~h}$, the mean contraction rate of the Embryo Rescue group was significantly reduced relative to the Complete Rescue group. The vast majority of Complete Rescue larvae was able to make full contractions at every time point in this experiment, but few larvae in the Embryo Rescue group made contractions at $40-48 \mathrm{~h}$, though all could respond to touch (see Materials and Methods). These data demonstrate a postembryonic requirement for Eaatl during larval locomotion.

\section{CNS development and apoptosis in Eaat1 mutants}

To investigate the effects of Eaat1 mutations on CNS development, we examined the integrity of well known CNS markers in late-stage embryos with immunohistochemistry, including one for a subset of VNC axons (Fas2), one for all VNC axons (HRP), and another to detect all lateral glia (Repo) (supplemental Fig. S6, available at www.jneurosci.org as supplemental material). We also examined CNS markers in L1 larvae, including Fas2 (Fig. $4 A, B$ ), the LG marker Naz (Fig. 4C,D), and the neuronal marker Eve (Fig. $4 E, F$ ). For all of these markers, there were no abnormalities observed in Eaat ${ }^{S M 2 / S M 2}$ mutants relative to heterozygous controls, suggesting that the CNS of Eaat1 mutants develops normally.

In the mammalian CNS, elevated levels of extracellular glutamate can lead to neuronal cell death from excitotoxicity (Sattler

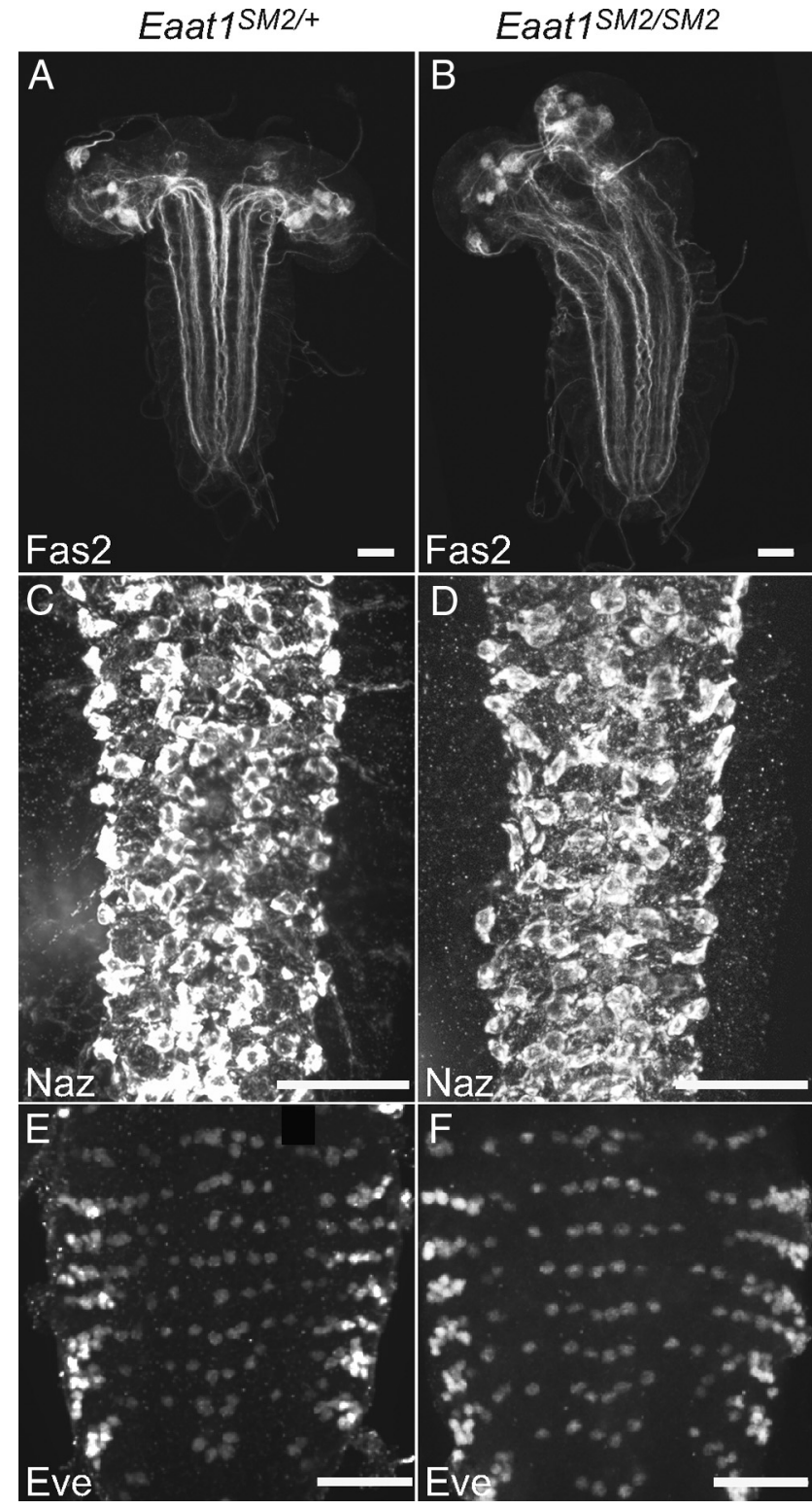

Figure 4. CNS markers are expressed normally in Eaat1 mutant L1 larvae. Eaat $7^{\mathrm{SM2} /+}$ het-

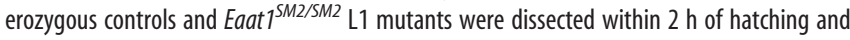
stained with immunohistochemistry for CNS markers. All panels show maximum projections of confocal images (Z-stacks) of the larval VNC. $A, B$, The axonal marker anti-Fas2. C, D, LG marker anti-Naz. $\boldsymbol{E}, \boldsymbol{F}$, Neuronal marker anti-Eve. No differences were observed between Eaat $7^{S M 2 /+}$ controls and Eaat ${ }^{\text {SM2/SM2 }}$ mutants, suggesting that the loss of Eaat1 does not cause gross developmental defects of the CNS and does not lead to excitoxicity-induced cell death. Scale bars, $20 \mu \mathrm{m}$.

and Tymianski, 2001). Similarly, loss of Eaat1 in Drosophila could lead to neurotoxicity and neuronal degeneration that could perhaps explain the larval crawling deficits. To examine this possibility further, we used immunostaining for cleaved caspase 3 and staining with acridine orange as two distinct methods to examine the occurrence of cell death in the CNS in Eaat1 mutants versus controls (Denton et al., 2008). However, neither approach revealed any observable increase in cells undergoing apoptosis (supplemental Fig. S7, available at www.jneurosci.org as supplemental material). In addition, we counted the number of Evepositive neurons per abdominal segment of the VNC (Fig. 4E, F). Eaat1 mutants had the same number of Eve-positive neurons per segment $(28.4 \pm 0.6, N=50$ segments $)$ as heterozygous controls 

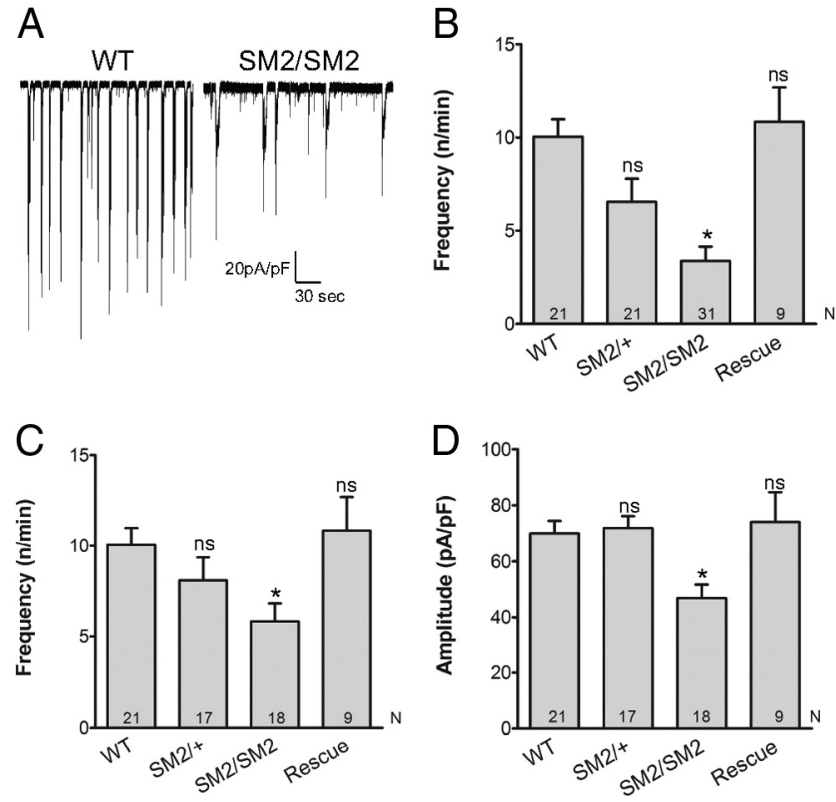

Figure 5. Excitation of motor neurons is reduced in Eaat 1 mutants. $A$, Representative wholecell voltage-clamp recordings of SRCs in motor neurons of WT and Eaat $7^{\text {SM2/SM2 }}$. B, C, SRC frequency is significantly decreased in Eaat $7^{5 M 2 / S M 2}$ mutants relative to WT controls, whether "silent" cells are included ( $\boldsymbol{B}$ ) or excluded ( $\boldsymbol{C}$. Values shown are mean \pm SEM. Asterisk indicates significance at $p<0.05$ (one-way ANOVA, Kruskal-Wallis test, Dunn's post hoc comparisons). $D$, SRC peak amplitude is also decreased in Eaat ${ }^{\text {SM2/SM2 }}$ mutants relative to WT controls. Asterisk indicates significance at $p<0.01$ (one-way ANOVA, Dunnett's post hoc test). Examples of individual peaks used to measure SRC amplitude are indicated in Figure $6 \mathrm{~A}$. Both SRC frequency and amplitude were rescued to WT levels by expression of GFP-tagged Eaat1 (genotype for rescue: Eaat1 ${ }^{\text {SM2 }}$, (G31235-Gal4; UAS-Eaat1::GFP). ns, Not significant.

(29.0 $\pm 0.5, N=65$ segments), providing additional evidence that the locomotor defects seen in newly hatched L1 larvae cannot be attributed to widespread neurotoxicity.

\section{Synaptic excitation of motor neurons is reduced in Eaat1 mutants}

Within the Drosophila VNC, the dorsal motor neurons known as aCC and RP2 are situated near the LG, and their electrophysiological properties can be measured in whole-cell patch-clamp recordings from acutely exposed cells in dissected first-instar larvae. These motor neurons exhibit large excitatory synaptic currents, termed SRCs (Rohrbough and Broadie, 2002), between 10 and 15 times every minute (Fig. 5A,B) (Baines et al., 2002). These currents, which result from endogenous activity of the locomoter central pattern generator circuit, evoke bursts of action potentials in motor neurons and underlie the patterned motor activity driving locomotion (Baines et al., 2001). SRCs arise from direct cholinergic input to these motor neurons although the identity of the premotor interneurons remains unknown (Baines et al., 1999). It is likely that glutamatergic neurotransmission modulates motor activity, since glutamate receptor GluR-IID null mutants exhibit a decrease in SRC frequency, associated with paralysis and death at late embryonic stages (Featherstone et al., 2005).

To determine whether Eaat1 influences the electrophysiological properties of aCC and RP2 motor neurons, we measured SRC frequency and amplitude in WT and Eaat $1^{S M 2 / S M 2}$ mutant larvae within 1-4 h after hatching (Fig. 5A). In WT larvae, these two neurons exhibit nearly identical properties; therefore, the data have been pooled from both. We found that both SRC frequency (Fig. 5B) and amplitude (Fig. 5D) were significantly decreased in the Eaat $1^{S M 2 / S M 2}$ mutants, with frequency being most severely
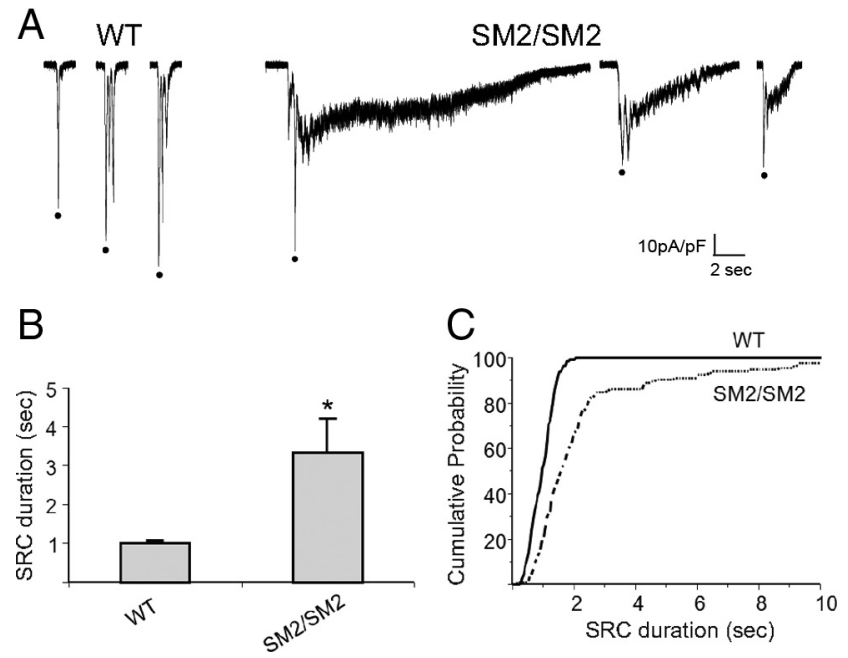

Figure 6. SRC duration is increased in Eaat1 mutants. $\boldsymbol{A}$, Representative SRC recordings in WT and Eaat ${ }^{\text {SM2/SM2 }}$. The individual peaks used to measure SRC amplitude are indicated by the dots. $B$, SRC duration is significantly increased in Eaat ${ }^{\text {SM2/SM2 }}$. Asterisk indicates significance at $p<0.01$ (two-tailed $t$ test, Mann-Whitney). C, Cumulative probability distribution of SRC duration in WT and Eaat $1^{\text {SM2/SM2 }}$ mutants.

affected. In fact, we observed the total absence of SRCs (during a 3 min recording period) in $42 \%$ of motor neurons (13/31). By comparison, the equivalent number of "silent" neurons in WT larvae was $0 \%(N=21)$. We consider silent cells to be extreme instances of reduced SRC frequency for the following reasons: first, some nonsilent cells had extremely low SRC frequency in Eaat ${ }^{S M 2 / S M 2}$ mutants; and second, silent and nonsilent cells were occasionally recorded from the same Eaat $1^{S M 2 / S M 2}$ mutant larva. When silent cells were excluded from the analysis, SRCs in Eaat ${ }^{S M 2 / S M 2}$ mutants remained significantly less frequent than controls (Fig. 5C). For peak amplitudes of SRCs observed in Eaat ${ }^{\text {SM2/SM2 }}$ mutants, the mean was reduced to 67 and $65 \%$, respectively, of WT and heterozygous controls (Fig. 5D).

In addition to reductions of frequency and amplitude, the kinetic properties of SRCs were also altered in Eaat1 mutants. In WT, single SRCs often exhibit multiple components, a finding that is consistent with asynchronous release from multiple premotor interneurons that provide synaptic drive to motor neurons (Baines et al., 2002). These component currents exhibit a fast onset and relatively fast offset, producing sharp events (Fig. $6 A)$. However, SRCs recorded in Eaat ${ }^{S M 2 / S M 2}$ mutant larvae often had an abnormally long decay (Fig. 6A), which increased mean SRC duration to $3.4 \pm 0.8 \mathrm{~s}$ from $1.0 \pm 0.1 \mathrm{~s}$ in wild-type controls (Fig. $6 \mathrm{~B}$ ). A plot of cumulative probability indicated that $\sim 40 \%$ of SRCs recorded in Eaat $1^{\text {SM2/SM2 }}$ were longer than the maximum duration seen in wild-type controls (Fig. 6C). Thus, in addition to regulating both amplitude and frequency of motor neuron synaptic drive, Eaat1 also contributes to the kinetics of SRCs.

The frequency and amplitude of SRCs in motor neurons of Eaat $1^{\text {SM2/SM2 }}$ mutants were fully rescued by CG31235-Gal4driven expression of a GFP-tagged Eaatl (Fig. 5B-D). Since CG31235-Gal4 is restricted to glia that reside in the CNS, and since dramatic SRC defects are readily recorded from the cell bodies of central motor neurons, we propose that the larval crawling defects observed in Eaat1 mutants are due, at least in part, to synaptic signaling defects within the CNS.

To determine whether the electrophysiological defects observed in Eaat1 mutants were consistent with a failure to trans- 
A
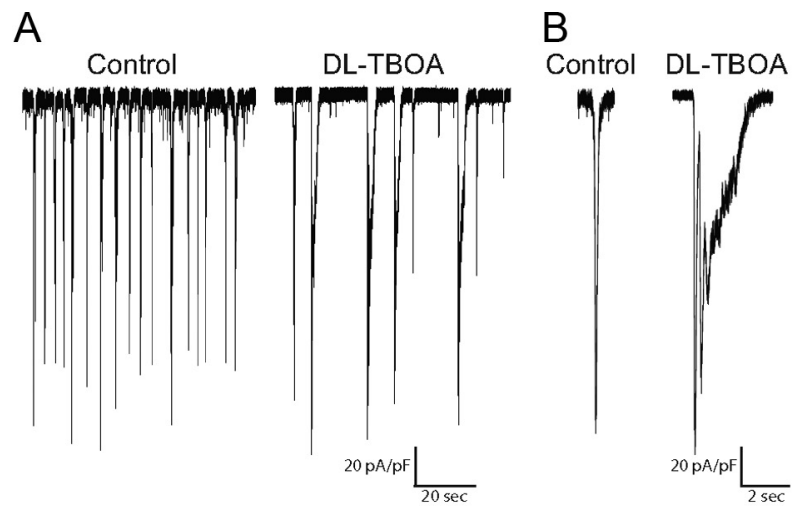

C
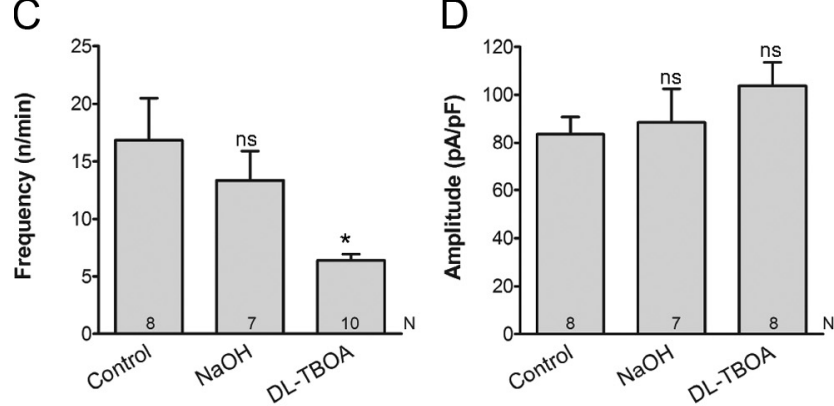

E

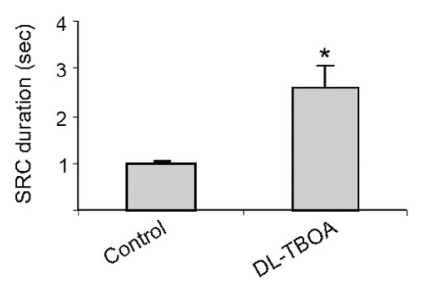

$\mathrm{F}$

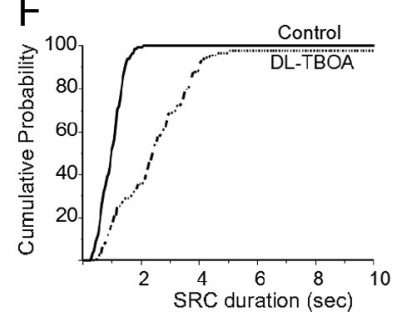

Figure 7. SRC frequency is reduced by the competitive EAAT antagonist DL-TBOA. $\boldsymbol{A}, \boldsymbol{B}$, Representative SRC recordings from WT motor neurons in the absence (Control or $\mathrm{NaOH}$ vehicle) or presence of bath-applied DL-TBOA. C, D, DL-TBOA reduces SRC frequency but does not influence amplitude. Values shown are mean \pm SEM. Asterisk indicates significance at $p<0.05$ (one-way ANOVA, Dunnett's post hoc test). $E$, SRC duration is increased in the presence of DL-TBOA treatment. Asterisk indicates significance at $p<0.01$ (two-tailed $t$ test, Mann-Whitney). $\boldsymbol{F}$, Cumulative probability distribution of SRC duration in WT in the absence (Control) or the presence of DL-TBOA. ns, Not significant.

port glutamate, we bath applied DL-TBOA, a competitive antagonist for EAATs (Shimamoto et al., 1998) (Fig. $7 A, B$ ) onto wild-type larvae. As in Eaat1 mutants, the mean SRC frequency was reduced; it reached only $48 \%$ of controls at the concentration tested $(300 \mu \mathrm{M})($ Fig. $7 C)$. There was no effect on amplitude (Fig. $7 D)$, but, as in Eaat1 mutants, the mean SRC duration was increased in the presence of DL-TBOA, from $1.0 \pm 0.1 \mathrm{~s}$ in controls to $2.6 \pm 0.5 \mathrm{~s}$ (Fig. $7 E$ ). More than $50 \%$ of SRCs recorded in the presence of DL-TBOA were longer than the maximum duration seen in controls (Fig. 7F).

Together, our results show that CNS glial expression of the glutamate transporter Eaat1 has a role in modulating patterned activity of motor neurons and support the idea that Eaat 1 expression in the anterior LG modulates glutamate neurotransmission.

\section{Discussion}

\section{The role of the glutamate transporter Eaat 1 in the Drosophila CNS}

We have created the first mutations in Drosophila Eaat1 as a means to better understand the importance of glutamate uptake for CNS development and function. Previous approaches using RNAi had uncovered no function for Eaat1 in larvae (Rival et al., 2004; Rival et al., 2006); this is likely due to insufficient knockdown at larval stages since here we found Eaat1 mutants have a severe crawling deficit. We narrowed the requirement for this glutamate transporter to a subpopulation of CNS glia and found that the crawling defect could be induced by conditional inactivation of Eaat1 after embryogenesis. Consistent with our observations that several immunohistochemical markers of neurons and glia were unaffected in Eaat1 mutants, these results indicate that the crawling deficit is not secondary to developmental defects. Furthermore, we found no evidence for widespread cell death in Eaat1 mutant larvae, suggesting that the crawling deficit is not a consequence of neurotoxic damage induced by excess glutamate.

Interestingly, Eaat1 mutant larvae withdrew and turned their heads normally in response to mechanical stimulus, raising the possibility that CNS neural circuits controlling rhythmic contractions required for larval crawling were specifically affected. The importance of glutamate-mediated neurotransmission in the CNS of Drosophila larvae has not been well characterized, though it has a well studied and essential role at the neuromuscular junction (DiAntonio, 2006). We have not specifically tested the function of neuromuscular synaptic transmission in Eaat1 mutants, but several lines of evidence indicate the crawling deficit is primarily due to a requirement for Eaat 1 within the CNS. First, electrophysiological recordings from motor neuron cell bodies within the CNS showed that Eaat1 mutants displayed decreased frequency of synaptic drive onto motor neurons. This mimics the effects we observed with acute pharmacological manipulations of glutamate transport, and resembles a published report on mutants of the AMPA-type glutamate receptor KaiRIA (also known as GluR-IID or brec) (Featherstone et al., 2005). Second, Eaat1 has been reported to be absent from neuromuscular junctions in larvae (Rival et al., 2006). Third, crawling was rescued with a Gal4 driver expressed exclusively in CNS glia. Together, our results lead us to propose that the crawling deficit in Eaat1 mutants is primarily due to a failure of specific glial cells in the CNS to efficiently remove excess glutamate from central synapses, leading to perturbed glutamatergic neurotransmission and reduced motor output. Since Drosophila motor neurons receive direct cholinergic input from premotor interneurons, we currently favor the idea that Eaat 1 acts to influence the patterning of rhythmic motor neuron activity by modulating glutamatergic synapses onto these premotor interneurons, or perhaps synapses further upstream in the circuitry (Baines et al., 2001; Featherstone et al., 2005).

Previous research has demonstrated that proteins essential for glutamatergic neurotransmission are expressed in the embryonic and larval CNS, such as VGlut (Mahr and Aberle, 2006; Daniels et al., 2008), and the ionotropic glutamate receptor subunits KaiRIA, GluR-IA, GluR-IB, Nmdar1, and Nmdar2 (Ultsch et al., 1992; Volkner et al., 2000; Featherstone et al., 2005). As noted above, AMPA-like receptors have already been implicated since KaiRIA (brec) mutants are paralyzed and have reduced SRC frequency (Featherstone et al., 2005). In addition, noncompetitive NMDA receptor antagonists have also been shown to inhibit rhythmic locomotor activity in larvae (Cattaert and Birman, 2001), but the role of these NMDA-like receptors in CNS motor circuitry remains to be tested by genetic means. The metabotropic glutamate receptor mGluRA is unlikely to be involved since null mutants are viable and have only mild defects of synaptic plasticity and morphology at neuromuscular syn- 
apses (Parmentier et al., 1996; Ramaekers et al., 2001; Bogdanik et al., 2004; Devaud et al., 2008).

One might predict that loss of glial glutamate uptake in Eaat1 mutants would elevate extracellular glutamate levels and lead to hyperactivity of glutamate receptors. However, SRC frequency is reduced by loss-of-function mutations of either Eaat1 or the KaiRIA (brec) glutamate receptor subunit, implying that Eaat1 mutants have reduced glutamatergic signaling instead. Perhaps this is due to receptor desensitization in the presence of excess glutamate. Eaat1 mutant larvae remain capable of some movement, while brec null mutants are completely paralyzed (Featherstone et al., 2005). This difference may reflect reduced glutamate neurotransmission resulting from excess receptor desensitization, versus the complete loss of neurotransmission in animals lacking an essential receptor subunit.

Currently, we cannot explain why pharmacological manipulations of glutamate transport affect SRC frequency and duration, while Eaat 1 mutations also affect SRC amplitude. It could reflect differences between acute, short-term treatment with DL-TBOA versus the genetic approach, in which loss of Eaat 1 function may lead to additional compensatory changes induced by prolonged increases of extracellular glutamate; it is conceivable that reduced SRC frequency is an acute and proximate effect of Eaat 1 mutations, and that the changes we observed in SRC peak amplitude are induced secondarily.

Our findings complement genetic studies in mice that are complicated by functional redundancy of the glial glutamate transporters GLAST and GLT-1. Mice singly mutant for GLAST or GLT-1 are viable (Tanaka et al., 1997; Watase et al., 1998), but double-knock-out mice die in utero and exhibit multiple proliferation and migration defects of stem cells and/or neurons in cortex, hippocampus, and olfactory bulb (Matsugami et al., 2006). As in Drosophila, glutamate neurotoxicity was not readily apparent in double-mutant mice, but the neuroanatomical defects observed in mice may reflect added importance for glutamate- and activity-dependent processes in the development of mammalian nervous systems. Knock-out mice for only GLAST fail complex motor tasks (Watase et al., 1998). This might reflect a role for this transporter in Bergmann glia of the cerebellum, where loss of GLAST is associated with inappropriate innervation and neurotransmission at glutamatergic synapses onto Purkinje neurons (Watase et al., 1998; Marcaggi et al., 2003; Takayasu et al., 2005, 2006). The acute role in crawling that we uncovered by conditional inactivation of Eaat 1 after embryogenesis supports the idea that, in Drosophila as in mice, glutamate transport strongly influences neurotransmission controlling motor function in vivo.

\section{Differentiation of functionally distinct glial cell subtypes}

We have found that the requirement for Eaat1 in locomotor behavior is limited to a subpopulation of glia marked by the CNSspecific driver CG31235-Gal4. At present, the tools available cannot distinguish the relative importance of glial cells located in the VNC versus the brain lobes. Nonetheless, Eaat1 is expressed in a limited subset of neuropil-associated glia in the VNC, including the anterior LG subtype, where it is coexpressed with the glutamate recycling enzyme Gs2 (Freeman et al., 2003; Thomas and van Meyel, 2007) and its expression is regulated by the glycosyltransferase Fng. Fng sensitizes the Notch receptor on the anterior LG to stimulation from developing axons bearing the Delta ligand and thereby promotes neuron-to-glial signaling during embryogenesis (Thomas and van Meyel, 2007). Anterior and posterior LG are derived from a common glioblast (Schmidt et al., 1997; Beckervordersandforth et al., 2008), and so, as a consequence of this interplay between neurons and glia, Fng provides a mechanism for the selective expression of Eaat 1 in the anterior LG subtype. Thus, Fng promotes the emergence of a functionally distinct glial cell subtype that can take up glutamate and has the potential to modulate neurotransmission at central synapses.

In vitro studies using cocultures of mammalian neurons and astrocytes have shown that factors secreted from neurons, and direct neuron-glial contact, can promote the expression of GLAST and/or GLT-1 in astrocytes (Swanson et al., 1997; Schlag et al., 1998; Munir et al., 2000; Zelenaia et al., 2000; Filosa et al., 2009; Yang et al., 2009). Studies in vivo provide evidence that the expression of glutamate transporters in the mammalian CNS is regulated by neuron-glial communication during development and also at mature stages. In the developing rodent cerebellum, for example, neuron-glial signaling through Notch and its ligand DNER regulates the maturation of Bergmann glia and promotes GLAST expression in these specialized astrocytes (Eiraku et al., 2005). Interestingly, the Fng ortholog Lunatic Fringe is expressed in Bergmann glia (Gong et al., 2003), but its involvement in neuron-glial interactions there remains unknown. In the mature hippocampus, direct neuron-glial contact and EphA4-ephrinA3 signaling regulate synaptic plasticity by controlling the expression of GLAST and GLT-1 and thereby regulating glutamate transport (Carmona et al., 2009; Filosa et al., 2009). Thus, mammals and insects both use neuron-glial communication to regulate glial glutamate transporter expression. The mechanism of regulation by Fng-mediated Notch signaling that we have discovered in flies may be conserved in the mammalian CNS, and the Eaat1 mutants described in this study provide an important model to study the molecular pathogenesis of CNS diseases in humans that result from dysregulation of glutamate transport.

\section{References}

Arama E, Steller H (2006) Detection of apoptosis by terminal deoxynucleotidyl transferase-mediated dUTP nick-end labeling and acridine orange in Drosophila embryos and adult male gonads. Nat Protoc 1:1725-1731.

Baines RA, Bate M (1998) Electrophysiological development of central neurons in the Drosophila embryo. J Neurosci 18:4673-4683.

Baines RA, Robinson SG, Fujioka M, Jaynes JB, Bate M (1999) Postsynaptic expression of tetanus toxin light chain blocks synaptogenesis in Drosophila. Curr Biol 9:1267-1270.

Baines RA, Uhler JP, Thompson A, Sweeney ST, Bate M (2001) Altered electrical properties in Drosophila neurons developing without synaptic transmission. J Neurosci 21:1523-1531.

Baines RA, Seugnet L, Thompson A, Salvaterra PM, Bate M (2002) Regulation of synaptic connectivity: levels of Fasciclin II influence synaptic growth in the Drosophila CNS. J Neurosci 22:6587-6595.

Barolo S, Carver LA, Posakony JW (2000) GFP and beta-galactosidase transformation vectors for promoter/enhancer analysis in Drosophila. Biotechniques 29:726, 728, 730:732.

Beart PM, O'Shea RD (2007) Transporters for L-glutamate: an update on their molecular pharmacology and pathological involvement. Br J Pharmacol 150:5-17.

Beckervordersandforth RM, Rickert C, Altenhein B, Technau GM (2008) Subtypes of glial cells in the Drosophila embryonic ventral nerve cord as related to lineage and gene expression. Mech Dev 125:542-557.

Besson MT, Soustelle L, Birman S (1999) Identification and structural characterization of two genes encoding glutamate transporter homologues differently expressed in the nervous system of Drosophila melanogaster. FEBS Lett 443:97-104.

Besson MT, Soustelle L, Birman S (2000) Selective high-affinity transport of aspartate by a Drosophila homologue of the excitatory amino-acid transporters. Curr Biol 10:207-210.

Besson MT, Ré DB, Moulin M, Birman S (2005) High affinity transport of taurine by the Drosophila aspartate transporter dEAAT2. J Biol Chem 280:6621-6626. 
Bogdanik L, Mohrmann R, Ramaekers A, Bockaert J, Grau Y, Broadie K, Parmentier ML (2004) The Drosophila metabotropic glutamate receptor DmGluRA regulates activity-dependent synaptic facilitation and fine synaptic morphology. J Neurosci 24:9105-9116.

Carhan A, Reeve S, Dee CT, Baines RA, Moffat KG (2004) Mutation in slowmo causes defects in Drosophila larval locomotor behaviour. Invert Neurosci 5:65-75.

Carmona MA, Murai KK, Wang L, Roberts AJ, Pasquale EB (2009) Glial ephrin-A3 regulates hippocampal dendritic spine morphology and glutamate transport. Proc Natl Acad Sci U S A 106:12524-12529.

Cattaert D, Birman S (2001) Blockade of the central generator of locomotor rhythm by noncompetitive NMDA receptor antagonists in Drosophila larvae. J Neurobiol 48:58-73.

Correia T, Papayannopoulos V, Panin V, Woronoff P, Jiang J, Vogt TF, Irvine KD (2003) Molecular genetic analysis of the glycosyltransferase Fringe in Drosophila. Proc Natl Acad Sci U S A 100:6404-6409.

Crisp S, Evers JF, Fiala A, Bate M (2008) The development of motor coordination in Drosophila embryos. Development 135:3707-3717.

Danbolt NC (2001) Glutamate uptake. Prog Neurobiol 65:1-105.

Daniels RW, Gelfand MV, Collins CA, DiAntonio A (2008) Visualizing glutamatergic cell bodies and synapses in Drosophila larval and adult CNS. J Comp Neurol 508:131-152.

Denton D, Mills K, Kumar S (2008) Methods and protocols for studying cell death in Drosophila. Methods Enzymol 446:17-37.

Devaud JM, Clouet-Redt C, Bockaert J, Grau Y, Parmentier ML (2008) Widespread brain distribution of the Drosophila metabotropic glutamate receptor. Neuroreport 19:367-371.

de Vries B, Mamsa H, Stam AH, Wan J, Bakker SL, Vanmolkot KR, Haan J, Terwindt GM, Boon EM, Howard BD, Frants RR, Baloh RW, Ferrari MD, Jen JC, van den Maagdenberg AM (2009) Episodic ataxia associated with EAAT1 mutation C186S affecting glutamate reuptake. Arch Neurol 66:97-101.

DiAntonio A (2006) Glutamate receptors at the Drosophila neuromuscular junction. Int Rev Neurobiol 75:165-179.

Eiraku M, Tohgo A, Ono K, Kaneko M, Fujishima K, Hirano T, Kengaku M (2005) DNER acts as a neuron-specific Notch ligand during Bergmann glial development. Nat Neurosci 8:873-880.

Featherstone DE, Rushton E, Rohrbough J, Liebl F, Karr J, Sheng Q, Rodesch CK, Broadie K (2005) An essential Drosophila glutamate receptor subunit that functions in both central neuropil and neuromuscular junction. J Neurosci 25:3199-3208.

Filosa A, Paixão S, Honsek SD, Carmona MA, Becker L, Feddersen B, Gaitanos L, Rudhard Y, Schoepfer R, Klopstock T, Kullander K, Rose CR, Pasquale EB, Klein R (2009) Neuron-glia communication via EphA4/ ephrin-A3 modulates LTP through glial glutamate transport. Nat Neurosci 12:1285-1292.

Freeman MR, Doherty J (2006) Glial cell biology in Drosophila and vertebrates. Trends Neurosci 29:82-90.

Freeman MR, Delrow J, Kim J, Johnson E, Doe CQ (2003) Unwrapping glial biology: $\mathrm{Gcm}$ target genes regulating glial development, diversification, and function. Neuron 38:567-580.

Go MJ, Eastman DS, Artavanis-Tsakonas S (1998) Cell proliferation control by Notch signaling in Drosophila development. Development 125: 2031-2040.

Gong S, Zheng C, Doughty ML, Losos K, Didkovsky N, Schambra UB, Nowak NJ, Joyner A, Leblanc G, Hatten ME, Heintz N (2003) A gene expression atlas of the central nervous system based on bacterial artificial chromosomes. Nature 425:917-925.

Griffiths RC, Benito-Sipos J, Fenton JC, Torroja L, Hidalgo A (2007) Two distinct mechanisms segregate Prospero in the longitudinal glia underlying the timing of interactions with axons. Neuron Glia Biol 3:75-88.

Hertz L, Dringen R, Schousboe A, Robinson SR (1999) Astrocytes: glutamate producers for neurons. J Neurosci Res 57:417-428.

Irvine KD, Wieschaus E (1994) fringe, a Boundary-specific signaling molecule, mediates interactions between dorsal and ventral cells during Drosophila wing development. Cell 79:595-606.

Jacobs JR, Hiromi Y, Patel NH, Goodman CS (1989) Lineage, migration, and morphogenesis of longitudinal glia in the Drosophila CNS as revealed by a molecular lineage marker. Neuron 2:1625-1631.

Jen JC, Wan J, Palos TP, Howard BD, Baloh RW (2005) Mutation in the glutamate transporter EAAT1 causes episodic ataxia, hemiplegia, and seizures. Neurology 65:529-534.
Kernan M, Cowan D, Zuker C (1994) Genetic dissection of mechanosensory transduction: mechanoreception-defective mutations of Drosophila. Neuron 12:1195-1206.

Lai EC, Bodner R, Kavaler J, Freschi G, Posakony JW (2000) Antagonism of notch signaling activity by members of a novel protein family encoded by the bearded and enhancer of split gene complexes. Development 127: 291-306.

Lee T, Luo L (1999) Mosaic analysis with a repressible cell marker for studies of gene function in neuronal morphogenesis. Neuron 22:451-461.

Mahr A, Aberle H (2006) The expression pattern of the Drosophila vesicular glutamate transporter: a marker protein for motoneurons and glutamatergic centers in the brain. Gene Expr Patterns 6:299-309.

Maier D, Nagel AC, Johannes B, Preiss A (1999) Subcellular localization of Hairless protein shows a major focus of activity within the nucleus. Mech Dev 89:195-199.

Marcaggi P, Billups D, Attwell D (2003) The role of glial glutamate transporters in maintaining the independent operation of juvenile mouse cerebellar parallel fibre synapses. J Physiol 552:89-107.

Matsugami TR, Tanemura K, Mieda M, Nakatomi R, Yamada K, Kondo T, Ogawa M, Obata K, Watanabe M, Hashikawa T, Tanaka K (2006) Indispensability of the glutamate transporters GLAST and GLT1 to brain development. Proc Natl Acad Sci U S A 103:12161-12166.

Matthias K, Kirchhoff F, Seifert G, Hüttmann K, Matyash M, Kettenmann H, Steinhäuser C (2003) Segregated expression of AMPA-type glutamate receptors and glutamate transporters defines distinct astrocyte populations in the mouse hippocampus. J Neurosci 23:1750-1758.

McGuire SE, Le PT, Osborn AJ, Matsumoto K, Davis RL (2003) Spatiotemporal rescue of memory dysfunction in Drosophila. Science 302:1765-1768.

Morel V, Lecourtois M, Massiani O, Maier D, Preiss A, Schweisguth F (2001) Transcriptional repression by suppressor of hairless involves the binding of a hairless-dCtBP complex in Drosophila. Curr Biol 11:789-792.

Munir M, Correale DM, Robinson MB (2000) Substrate-induced upregulation of $\mathrm{Na}(+)$-dependent glutamate transport activity. Neurochem Int 37:147-162.

Murai KK, Van Meyel DJ (2007) Neuron glial communication at synapses: insights from vertebrates and invertebrates. Neuroscientist 13:657-666.

Parmentier ML, Pin JP, Bockaert J, Grau Y (1996) Cloning and functional expression of a Drosophila metabotropic glutamate receptor expressed in the embryonic CNS. J Neurosci 16:6687-6694.

Ramaekers A, Parmentier ML, Lasnier C, Bockaert J, Grau Y (2001) Distribution of metabotropic glutamate receptor DmGlu-A in Drosophila melanogaster central nervous system. J Comp Neurol 438:213-225.

Rival T, Soustelle L, Strambi C, Besson MT, Iché M, Birman S (2004) Decreasing glutamate buffering capacity triggers oxidative stress and neuropil degeneration in the Drosophila brain. Curr Biol 14:599-605.

Rival T, Soustelle L, Cattaert D, Strambi C, Iché M, Birman S (2006) Physiological requirement for the glutamate transporter dEAAT1 at the adult Drosophila neuromuscular junction. J Neurobiol 66:1061-1074.

Rohrbough J, Broadie K (2002) Electrophysiological analysis of synaptic transmission in central neurons of Drosophila larvae. J Neurophysiol $88: 847-860$.

Sattler R, Tymianski M (2001) Molecular mechanisms of glutamate receptor-mediated excitotoxic neuronal cell death. Mol Neurobiol 24:107-129.

Schlag BD, Vondrasek JR, Munir M, Kalandadze A, Zelenaia OA, Rothstein JD, Robinson MB (1998) Regulation of the glial $\mathrm{Na}+$-dependent glutamate transporters by cyclic AMP analogs and neurons. Mol Pharmacol 53:355-369.

Schmidt H, Rickert C, Bossing T, Vef O, Urban J, Technau GM (1997) The embryonic central nervous system lineages of Drosophila melanogaster. II. Neuroblast lineages derived from the dorsal part of the neuroectoderm. Dev Biol 189:186-204.

Sepp KJ, Auld VJ (2003) Reciprocal interactions between neurons and glia are required for Drosophila peripheral nervous system development J Neurosci 23:8221-8230.

Shimamoto K, Lebrun B, Yasuda-Kamatani Y, Sakaitani M, Shigeri Y, Yumoto N, Nakajima T (1998) DL-threo-beta-benzyloxyaspartate, a potent blocker of excitatory amino acid transporters. Mol Pharmacol 53:195-201.

Shishido E, Ono N, Kojima T, Saigo K (1997) Requirements of DFR1/ Heartless, a mesoderm-specific Drosophila FGF-receptor, for the forma- 
tion of heart, visceral and somatic muscles, and ensheathing of longitudinal axon tracts in CNS. Development 124:2119-2128.

Soustelle L, Besson MT, Rival T, Birman S (2002) Terminal glial differentiation involves regulated expression of the excitatory amino acid transporters in the Drosophila embryonic CNS. Dev Biol 248:294-306.

Stacey SM, Thomas GB, Labbé A, Van Meyel DJ (2007) Longitudinal glia in the fly CNS: pushing the envelope on glial diversity and neuron-glial interactions. Neuron Glia Biol 3:27-33.

Swanson RA, Liu J, Miller JW, Rothstein JD, Farrell K, Stein BA, Longuemare MC (1997) Neuronal regulation of glutamate transporter subtype expression in astrocytes. J Neurosci 17:932-940.

Takayasu Y, Iino M, Kakegawa W, Maeno H, Watase K, Wada K, Yanagihara D, Miyazaki T, Komine O, Watanabe M, Tanaka K, Ozawa S (2005) Differential roles of glial and neuronal glutamate transporters in Purkinje cell synapses. J Neurosci 25:8788-8793.

Takayasu Y, Iino M, Shimamoto K, Tanaka K, Ozawa S (2006) Glial glutamate transporters maintain one-to-one relationship at the climbing fiberPurkinje cell synapse by preventing glutamate spillover. J Neurosci 26: 6563-6572.

Tanaka K, Watase K, Manabe T, Yamada K, Watanabe M, Takahashi K, Iwama H, Nishikawa T, Ichihara N, Kikuchi T, Okuyama S, Kawashima N, Hori S, Takimoto M, Wada K (1997) Epilepsy and exacerbation of brain injury in mice lacking the glutamate transporter GLT-1. Science 276:1699-1702.

Thomas GB, van Meyel DJ (2007) The glycosyltransferase Fringe promotes Delta-Notch signaling between neurons and glia, and is required for subtype-specific glial gene expression. Development 134:591-600.
Ultsch A, Schuster CM, Laube B, Schloss P, Schmitt B, Betz H (1992) Glutamate receptors of Drosophila melanogaster: cloning of a kainateselective subunit expressed in the central nervous system. Proc Natl Acad Sci U S A 89:10484-10488.

van Meyel DJ, Thomas JB, Agulnick AD (2003) Ssdp proteins bind to LIMinteracting co-factors and regulate the activity of LIM-homeodomain protein complexes in vivo. Development 130:1915-1925.

Völkner M, Lenz-Böhme B, Betz H, Schmitt B (2000) Novel CNS glutamate receptor subunit genes of Drosophila melanogaster. J Neurochem 75:1791-1799.

von Hilchen CM, Hein I, Technau GM, Altenhein B (2010) Netrins guide migration of distinct glial cells in the Drosophila embryo. Development 137:1251-1262.

Watase K, Hashimoto K, Kano M, Yamada K, Watanabe M, Inoue Y, Okuyama S, Sakagawa T, Ogawa S, Kawashima N, Hori S, Takimoto M, Wada K, Tanaka K (1998) Motor discoordination and increased susceptibility to cerebellar injury in GLAST mutant mice. Eur J Neurosci 10:976-988.

Yang Y, Gozen O, Watkins A, Lorenzini I, Lepore A, Gao Y, Vidensky S, Brennan J, Poulsen D, Won Park J, Li Jeon N, Robinson MB, Rothstein JD (2009) Presynaptic regulation of astroglial excitatory neurotransmitter transporter GLT1. Neuron 61:880-894.

Zelenaia O, Schlag BD, Gochenauer GE, Ganel R, Song W, Beesley JS, Grinspan JB, Rothstein JD, Robinson MB (2000) Epidermal growth factor receptor agonists increase expression of glutamate transporter GLT-1 in astrocytes through pathways dependent on phosphatidylinositol 3-kinase and transcription factor NF-kappaB. Mol Pharmacol 57:667-678. 